Historic, Archive Document

Do not assume content reflects current scientific knowledge, policies, or practices. 



\title{
Price List of Nursery Stock
}

OF THE

\section{American Nursery Company}

\section{Singer Building, New York City}

\section{EIGHT HUNDRED \\ ACRES}

\author{
NURSERIES \\ Flushing, L. I. \\ Albertson, L. I. \\ Searingtown, L. I. \\ Springfield, N. J.
}

\section{GUARANTEE-}

WE GUARANTEE all our stock true to name and in good condition on leaving our hands, but we do not guarantee it to grow under all conditions, nor do we make up any loss whatever due to climate effects, or after culture on the part of the purchaser. In case any mistake should happen in the naming of a plant, we will replace it with the true variety as ordered, but we are not liable to more than its original invoice value in refunding money. 



\section{NOTICE TO CUSTOMERS}

We would say to those unacquainted with us that we have 800 acres devoted to the business; our methods of growing and handling stock are up to date. We solicit correspondence and personal inspection of our stock, confident that it will compare favorably with the best.

REFERENCES-For those who do not know us we refer to any leading nurserymen in the United States, Dun and Bradstreet's Mercantile Agencies.

PRICES-This list supersedes all previous editions. The prices are for the quantities named: 25 and 5 will be furnished at the 100 and 10 rates respectively. We have tried to make our prices as low as we think well-grown stock should be sold. We expect, however, to sell on the market and meet reliable competition.

TERMS-We are glad to grant 30 to 60 days' time for payment of bills to all responsible parties, but parties we do not know, who have no commercial rating, must send remittance with order, or a guarantee signed by some bank or someone of known responsibility to be sure of securing goods selected. If references are sent, we will confer with them and after hearing favorably, if goods selected are still unsold, we will enter them as requested, but we will not enter orders or ship goods until we know the customer is responsible.

We will not hold goods on inquiries.

Trees selected by customers at the nursery will be charged for in accordance with their value.

PACKING-This is done in boxes and bales for which a charge is made sufficient to remunerate for the cost of material only. Trees packed in bulk at no extra charge.

COMPLAINTS-Should there be any reason for complaint we must be notified on receipt of goods, otherwise we cannot entertain it. We make it a point to give all reasonable claims proper consideration.

ORDERS-Are accepted upon condition that they shall be void should any injury befall the stock from frost, or other cause over which we have no control.

VARIETIES-We reserve the right to select the varieties, but will give the purchaser his choice as far as is consistent with our stock. In ordering please state whether or not substitution will be permitted in case we should be short of any sort wanted.

GUARANTEE OF GENUINENESS-While we exercise the greatest care to have all our trees and plants genuine and reliable, and hold ourselves prepared to replace on proper proof, all trees that may prove untrue, we do not give any warranty expressed or implied; and in case of any error on our part, it is mutually agreed between the purchaser and ourselves that we shall not at any time be held responsible for a greater amount than the original price of the stock.

We guarantee all our stock to be in good condition on leaving our hands, but we do not guarantee it to grow under all conditions, nor do we make up any loss whatever due to climate effects, or after culture on the part of the purchaser and the risks of transportation are to be borne by purchaser. When losses occur by detention, claims should be at once made on forwarders. 



\section{ORNAMENTAL DEPARTMENT}

\section{DECIDUOUS TREES}

\begin{tabular}{|c|c|c|c|}
\hline & Tel. Code & Each & Per 10 \\
\hline ACER CAMPESTRE (BUSH) & $2-3 \mathrm{ft} \ldots$. & $\$ 1.00$ & $\$ 9.50$ \\
\hline - DASYCARPUM & ....gadfly & 1.25 & 10.00 \\
\hline Silver Maple & $8-10$ t., $1 \frac{1}{4}$ in. . gadder & 1.50 & 12.00 \\
\hline & $10-11 \mathrm{ft} ., 11 / 2$ in. .gadding & 1.75 & 15.00 \\
\hline & $10-12 \mathrm{ft} ., 13 / 4$ in. . gaddingly & 2.25 & 18.00 \\
\hline & $12-13 \mathrm{ft} ., 2$ in . gaelic .... & 3.00 & 25.00 \\
\hline & 13-14 ft., $21 / 2$ in. gaffle . & 3.75 & 35.00 \\
\hline & 14-15 ft., 3 in. . . gafty & 5.00 & 45.00 \\
\hline & $14-15 \mathrm{ft} ., 3 \frac{1}{2}-4$ in.gag. & 6.00 & \\
\hline - - WIERII & $8-10 \mathrm{ft},, 11 / 4$ in . gaggling . . & 2.00 & 17.50 \\
\hline Wier's Cut Leaf & $10-11 \mathrm{ft}$., $11 \frac{1}{2}$ in. . gaily ..... & 2.25 & 20.00 \\
\hline & $11-12 \mathrm{ft} ., 13 / 4$ in . gain $\ldots \ldots$ & 2.50 & 22.50 \\
\hline & $12-13 \mathrm{ft}$., 2 in . gainage ... & 3.50 & 32.50 \\
\hline & $12-15 \mathrm{ft} ., 21 / 2$ in. . gainer & 5.00 & 45.00 \\
\hline & $12-15 \mathrm{ft} ., 3$ in . gainful. ... & 7.00 & \\
\hline - NEGUNDO. & . $8-10 \mathrm{ft} ., 11 / 4$ in . gainless ... & 1.25 & 10.00 \\
\hline Ash Leaf Maple & $10-11 \mathrm{ft} ., 1 \frac{1}{2}$ in. . gainly & 1.75 & 15.00 \\
\hline & $11-12 \mathrm{ft} ., 13 \% 4$ in . gainsay ... & 2.00 & 17.50 \\
\hline & $12-13 \mathrm{ft}$., 2 in . gainsayer & 2.50 & 22.50 \\
\hline & $12-14 \mathrm{ft},, 21 / 2$ in. . garish ... & 3.50 & 32.50 \\
\hline — PLATANOIDES. & . $8-10 \mathrm{ft} ., 11 \frac{1}{4}$ in . gaiter.... & 200 & 17.50 \\
\hline NORWAy MaPLE & $10-11 \mathrm{ft} ., 1 \frac{1}{2}$ in. . gala . & 2.50 & 22.50 \\
\hline & $11-12 \mathrm{ft} ., 13 \frac{4}{4}$ in. . galaxy & 3.00 & 27.50 \\
\hline & $12-13 \mathrm{ft} ., 2-2 \frac{1}{2}$ in galban ... & 4.00 & 37.50 \\
\hline & $13-14 \mathrm{ft}$., $21 / 2$ in. . galeas ... & 5.00 & 45.50 \\
\hline & $14-15 \mathrm{ft} ., 3$ in. . . galeate ... & 7.50 & 72.50 \\
\hline & $14-15 \mathrm{ft},, 31 / 2 \mathrm{i}$. . galeated . & 10.00 & 95.00 \\
\hline & 4 in . galenic... & 18.00 & 125.00 \\
\hline - - SCHWEDLERII & $8-10 \mathrm{ft} . \ldots \ldots \ldots$ galenical .. & 3.50 & \\
\hline $\begin{array}{l}\text { SCHWEdLER's PURPLE } \\
\text { - PSEUDO PLATANUS }\end{array}$ & & & \\
\hline - PSEUDO PLATANUS & $8-10 \mathrm{ft} ., 11 / 2$ in. .gall & 2.50 & 22.00 \\
\hline Sycamore Maple & 9-11 ft., $13 / 4$ in. .gallant. . & 3.00 & 28.00 \\
\hline & $10-12 \mathrm{ft} ., 2$ in . gallantly & 3.50 & 32.50 \\
\hline & 11-13 ft., $21 / 2$ in. . gallantry & 5.00 & 47.50 \\
\hline & 12-14 ft., 3 in . gallate & 7.00 & 67.50 \\
\hline & $13-14 \mathrm{ft}$., $3 \frac{1}{2}$ in. .galleries... & 10.00 & \\
\hline - RUBRUM & $8-10 \mathrm{ft}$., $1 \frac{1}{4}$ in. . gallery & 3.00 & 25.00 \\
\hline Red or Scarlet Maple & 10-12 ft., $1 \frac{1}{2}$ in. galley . & 3.25 & 30.00 \\
\hline & $12-13 \mathrm{ft}$., $13 / 4$ in. . gallic & 3.75 & 35.00 \\
\hline & 13-14 ft., 2 in. .gallican .. & 5.00 & 47.50 \\
\hline & $13-14 \mathrm{ft} ., 21 / 2$ in. . gallivant & 6.50 & 60.00 \\
\hline — SACCHARINUM & .... galloon .. & 2.00 & 18.00 \\
\hline Sugar Maple & $9-11 \mathrm{ft} ., 11 / 4$ in. . gallooned & 2.50 & 22.00 \\
\hline & $10-12 \mathrm{ft} ., 11 / 2$ in . gallop & 2.75 & 25.00 \\
\hline & $11-13 \mathrm{ft} ., 13 \frac{1}{4}$ in. . gallopade . & 3.50 & 32.00 \\
\hline & $12-14 \mathrm{ft} ., 2$ in. .galloper . . & 4.50 & 40.00 \\
\hline & $14-16 \mathrm{ft}$., $21 / 2$ in . . galls & 5.50 & 50.00 \\
\hline & $14-16 \mathrm{ft} ., 3$ in. .galvanized & 7.00 & 65.00 \\
\hline - STRIATUM & $4-6$ & 2.00 & \\
\hline AESCULUS HIPPOCASTANUM & $8 \mathrm{ft} ., 1 \frac{1}{4}$ in . . . . gambler... & 2.25 & 20.00 \\
\hline EUROPEAN OR COMMON & $8-10 \mathrm{ft} ., 1 \frac{1}{2}$ in . gambling. . & 2.75 & 25.00 \\
\hline HoRSECHESTNUT & $8-10 \mathrm{ft} ., 13 \frac{1}{4}$ in. .game & 3.50 & 32.50 \\
\hline & $10-12 \mathrm{ft} ., 2$ in. .gameful & 4.00 & 37.50 \\
\hline & 10-12 ft., $21 / 2$ in. .gamesome & 6.00 & 55.00 \\
\hline & 10-12 ft., 3 in. .gamester. & 7.00 & 67.50 \\
\hline & $12-13 \mathrm{ft}$., $3 \frac{1}{2}-4$ in.gammon .. & 10.00 & 75.00 \\
\hline & $12-13 \mathrm{ft} ., 4$ in. .gamut.... & 25.00 & 225.00 \\
\hline
\end{tabular}

(5) 
ACER-Continued

- - RUBICUNDA

Red Flowering Horsechestyut

- - ALBA FLORA PLENA

DBl. White Flowerivg

- (PAVIA) MACROSTACHIA, see shrubs

ALNUS GLUTINOSA ............

\section{AMYGDALUS PERSICA}

Flowerixg PEACH

Red and White

ANDROMEDA (See Shrubs)

ARALIA (See Shrubs)

BETULA ALBA

European White Birch

\section{- LACINIATA}

Weeping Cut Leaf

\section{- - PEND. YOUNGII \\ (Weeping) \\ - - PYRAMIDALIS}

- LENTA

SWEET Birch

- LUTEA.

YELLOW BIRCH

- NIGRA

Black or River Birch

- PAPYRACEA

Paper or CaNoe Birch

CARPINUS AMERICANA

AMericay Hornbeam

\section{- BETULUS}

CATALPA BIGNOIDES

INDIAN BEAN

- BUNGEI Umbrella Catalpa

Medium heads .............. 11/4 in. stems ....gasp .... 2.00

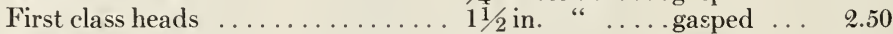

Extra large heads............ 13/4 in. " .....gasping ... 3.00

" " " $\ldots \ldots \ldots \ldots \ldots \ldots 2$ in. " ..... gassy ... 4.00

CELTIS OCCIDENTÄIS (See Shrubs)

\section{CEPHALANTHUS (See Shrubs)}

\section{CERASUS}

- JAP. ROSEA PENDULA ......... 3- $4 \mathrm{ft} \ldots \ldots \ldots$ gathered . . 1.75

WeEPING Japan CherRy

Standard or Pyramidal

- PENDULA, double flowering

- SEROTINA

$$
\text { t- } 5 \mathrm{ft} \ldots \ldots \ldots \text {...... gather... }
$$

$5-6 \mathrm{ft} \ldots \ldots \ldots$. gathering .

$6-8 \mathrm{ft} \ldots \ldots$. . gather .... 4.00

$3-4 \mathrm{ft} \ldots \ldots$.... gaud .... 2.00

$4-6 \mathrm{ft} \ldots \ldots \ldots$ gaudily ... 2.75

t- $6 \mathrm{ft} \ldots \ldots \ldots$. gaudery... 1.50

WiLd Black CHERRY

$6-8 \mathrm{ft} \ldots \ldots$....gaudful ... 1.75

- SINENSIS FL. PL. - Red ANd White $t-6 \mathrm{ft} \ldots \ldots \ldots$ gaudless ... 2.50 Chinese Dbl. Fla.
$\$ 12.50$

15.00

6.50

8.50

10.00

8.50

8.50

12.50

17.50

27.50

12.00

16.00

2000

25.00

30.00

20.00

12.50

17.50

22.50

7.50

22.50

27.50

17.50

22.50

27.50

22.50

8.50

12.50

17.50

9.00

15.00

17.50

20.00

12.00

15.00 
CERASUS-Continued

Tel. Code Each Per 10

- SPECTABILIS ROSEA

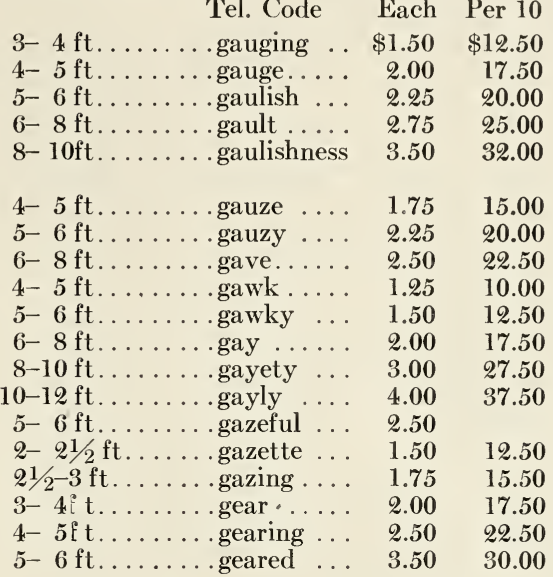

CRATAEGUS, Thorn

- ARNOLDIANA.

\begin{tabular}{|c|c|c|}
\hline ..... gelder & 1.00 & 7.50 \\
\hline $4-5 \mathrm{ft} \ldots \ldots \ldots$ gelding & 1.25 & 10.00 \\
\hline $3-4 \mathrm{ft} \ldots \ldots \ldots$ gelded .... & 1.25 & 10.00 \\
\hline $4-5 \mathrm{ft} \ldots \ldots \ldots$ geld $\ldots \ldots$ & 1.50 & 12.50 \\
\hline $3-4 \mathrm{ft} \ldots \ldots \ldots$ gelt & 1.00 & 8.50 \\
\hline $4-5 \mathrm{ft} \ldots \ldots$. . gelid & 1.25 & \\
\hline $3-4 \mathrm{ft} \ldots \ldots$. . gelidness . & 1.00 & 8.50 \\
\hline $4-6 \mathrm{ft} \ldots \ldots \ldots$ gemaric & 1.50 & 12.50 \\
\hline $2-3 \mathrm{ft} \ldots \ldots \ldots$ gemination & .75 & 6.00 \\
\hline $3-4 \mathrm{ft} \ldots \ldots$....geminous . & 1.00 & 8.50 \\
\hline $4-5 \mathrm{ft} \ldots \ldots \ldots$ gemmary . & 1.50 & 12.50 \\
\hline $3-4 \mathrm{ft} \ldots \ldots$.... gemmated. & 1.00 & \\
\hline $3-4 \mathrm{ft} \ldots . . \ldots$ gemmy ... & 1.00 & 8.50 \\
\hline $4-5 \mathrm{ft} \ldots \ldots \ldots$ gender $\ldots$ & 1.50 & 12.50 \\
\hline $3-4 \mathrm{ft} \ldots \ldots$. genealogist & 1.00 & \\
\hline $4-5 \mathrm{ft} \ldots \ldots \ldots$ generality & 1.25 & \\
\hline $4-5 \mathrm{ft} \ldots \ldots \ldots$ generally & 1.25 & \\
\hline $4-6 \mathrm{ft} \ldots \ldots \ldots$ generalize . & 1.00 & 8.50 \\
\hline $6-8 \mathrm{ft} \ldots \ldots \ldots$ general ... & 1.50 & 12.50 \\
\hline $3-4 \mathrm{ft} \ldots \ldots$. . generate & 2.00 & 17.50 \\
\hline $4-5 \mathrm{ft} \ldots \ldots \ldots$ generated . & 2.25 & 20.00 \\
\hline $5-6 \mathrm{ft} \ldots \ldots \ldots$ generating & 2.50 & 22.50 \\
\hline $6-7 \mathrm{ft} \ldots \ldots \ldots$ generation & 3.50 & \\
\hline $3-4 \mathrm{ft} \ldots \ldots \ldots$ generosity & 2.00 & 17.50 \\
\hline ... generous. . & 2.50 & 22.50 \\
\hline ...generously & 3.00 & 27.50 \\
\hline ...genial .... & 2.00 & 19.00 \\
\hline . . genesis. ... & 2.50 & \\
\hline $5-6 \mathrm{ft} \ldots \ldots$ & 3.00 & \\
\hline $6-8 \mathrm{ft} \ldots \ldots \ldots$ genteel ... & 4.00 & \\
\hline $8-10 \mathrm{ft} \ldots . .$. . genteely . . & 5.00 & \\
\hline $10-12 \mathrm{ft} \ldots \ldots$. . gentile $\ldots$ & 7.50 & \\
\hline $3-4 \mathrm{ft} \ldots \ldots \ldots$ gentility . . & 2.50 & \\
\hline $4-5 \mathrm{ft} \ldots \ldots \ldots$ gentilize . . & 3.50 & \\
\hline $5-6 \mathrm{ft} \ldots \ldots$ gentle $\ldots$ & 5.00 & \\
\hline ... gentleman. & 7.00 & \\
\hline$\ldots$ gently .... & 3.75 & \\
\hline ...gentry. & 4.50 & \\
\hline ...genuine . & 2.50 & \\
\hline . genuinly. & 3.50 & \\
\hline$\ldots$ genus $\ldots .$. & 4.00 & \\
\hline $6-8 \mathrm{ft} \ldots \ldots$. . geography. & 5.00 & \\
\hline
\end{tabular}

7.50

- CARRIERI

$\ldots \ldots \ldots \ldots$

COCCINEA

White Thorn

- CORDATA

Washington ThORN

- CRUS GALLI.

Cockspur Thorn

- MOLLE

OXYCANTHA, Dbi. Flg.

pink, red and white

- TOMENTOSUM

........

CYTISSUS LABURNUM

DYOSPYRUS VIRGINIANA

Persimmon

EUONYMUS (See Shrubs)

FAGUS FERRUGINEA.
American Beech

- SYLVATICA

ENGLish BeEch

(n...........

- HETEROPHYLLA

Fern or Cut Lvd. Bee.

- - PENDUla

WEEPING BEECH

\section{- - PURPUREA}

Purple Copper Beech

- - PENDULA

Weeping Purple Beech

- - - RIVERSI

River's Purple BeEch 


\section{FRAXINUS AMERICANA}

White AsH

- VIRIDIS

\section{GYMNOCLADUS}

KeNtuCky Coffee Tree

HALESIA (See Shrubs)

KOELREUTERIA

- PANICUlatA-VARNish Tree … LARIX EUROPEA-Etropeax Larch

- LEPTOLEPIS

JAPAN LARCH

\section{LIQUIDAMBER STYRACIFLUA}

SweEt GuM

LIRIODENDRON TULIPFERA

Trlip Tree

MAGNOLIA ACUMINATA

Cucumber Tree

- ALBA SUPERBA, with ball

- GLAUCA, Sweet BA

\section{— LENNEI \& SOULANGIANA}

- STELLATA HALLEANA, with ball . StarRy Magnolia

- TRIPETELA

Umbrella Tree

\section{MALUS CORONARIUS \\ - FLORIBUNDA and ATROSANGUINEA}

\section{- IOENSIS}

Bechtel's Crab

- PaRkMan's, Parkman's Crab

MORUS TARTARICA PENDULA.

Tea's Weeping Mulberry

Medium heads glade ..... 2.75

First class head gladden .

Extra heads

Double extra hear

OSTRYA VIRGINICA

PAULOWNIA IMPERIALIS Empress Tree

PHELLODENDRON AMURENSE Chinese Cork Tree
Tel. Code

$8-10 \mathrm{ft} ., 11 / 4$ in. geometer $10-12 \mathrm{ft} ., 11 / 2$ in. .geometric $10-12 \mathrm{ft} ., 13 / 4$ in . geometrize 12-13 ft., 2 in. geometry 8-10 ft., $11 / 2$ in. germinal . . $10-12 \mathrm{ft} ., 13 / 4$ in. .germinate 12-13 ft., 2 in. germ .... 3- $4 \mathrm{ft} \ldots . .$. . german ... $t-5 \mathrm{ft} . . . .$. gest ......... $3-4 \mathrm{ft} \ldots \ldots$. . gester . . .

$4-6 \mathrm{ft} \ldots \ldots$. . gestic ... 1.00

g- $3 \mathrm{ft} \ldots . .$. gestor ... $\quad 1.00$

t- $6 \mathrm{ft} \ldots \ldots$. gesture ... $\quad \mathcal{2 . 0 0}$

(6- $8 \mathrm{ft} \ldots \ldots$. . geyser ... $\quad 2.50$

$8-10 \mathrm{ft} \ldots . .$. ghast .... 3.00

10-12 ft ....... ghastful. . 4.00

t- $6 \mathrm{ft} \ldots \ldots$. . ghastly ... 2.20

$6-8 \mathrm{ft} \ldots \ldots \ldots$ ghost ..... 2.50

$8-10 \mathrm{ft} \ldots \ldots$. . . ghostless . 3.00

$4-6 \mathrm{ft} \ldots \ldots$. . ghostlike . $\quad 1.00$

6- $8 \mathrm{ft} \ldots . .$. . ghostly ... 1.75

$8-10 \mathrm{ft} \ldots \ldots$. giant ..... 2.50

$10-12 \mathrm{ft} \ldots . .$. . gibber ... $\quad 3.00$

$4-6 \mathrm{ft} \ldots \ldots$. . gibbet .... 1.25

$6-7 \mathrm{ft} \ldots \ldots$. . gibe ..... 2.00

$8 \mathrm{ft} \ldots \ldots . . .$. gibing ... $\quad 3.00$

$2-3 \mathrm{ft} \ldots \ldots . .$. gibling ... 5.00

$3-4 \mathrm{ft} \ldots \ldots$.... giblet ... 6.50

\&- $3 \mathrm{ft} \ldots \ldots \ldots$ gift ....... 1.50

$3-4 \mathrm{ft} \ldots . .$. gifted.... 2.00

t- $5 \mathrm{ft} \ldots . .$. giggle.... $\quad 2.50$

$2 \mathrm{ft} \ldots \ldots \ldots$. . . gilded .... 3.50

$3-4 \mathrm{ft} \ldots \ldots$. . . guilty . . . 5.00

$2-21 / 2 \mathrm{ft} \ldots \ldots$ gird ...... 5.50

$3 \mathrm{ft} \ldots \ldots \ldots$. . . . girded ... 6.50

+ $6 \mathrm{ft} \ldots \ldots$.... girdled... $\quad 2.25$

$6-8 \mathrm{ft} \ldots \ldots \ldots$ girdler ... 3.00

$8-10 \mathrm{ft} \ldots . .$. girdle .... 3.50

$12 \mathrm{ft} \ldots \ldots \ldots$. . . . . . 4.00

$3-4 \mathrm{ft} \ldots \ldots$... girlhood .. 1.50

$3-4 \mathrm{ft} \ldots \ldots \ldots$ girlish .... 1.00

$4-5 \mathrm{ft} \ldots \ldots$. girlishly... 1.25

$5-6 \mathrm{ft} \ldots \ldots$. girlishness $\quad 1.50$

$2-21 / 2 \mathrm{ft} \ldots \ldots$ give ..... $\quad .75$

$21 / 2-3 \mathrm{ft} \ldots \ldots$ given $\quad 1.00$

$3-4 \mathrm{ft} \ldots \ldots$ giver .......... 1.25

$4-5 \mathrm{ft} \ldots \ldots$. . giving ... 1.50

$3-4 \mathrm{ft} \ldots \ldots$. . gizzard ... 1.50
12.50

17.50

20.00

27.50

37.50

17.50

22.50

27.50

8.50

15.00

22.00

12.50

17.50

20.00

27.50

30.00

17.50

20.00

27.50 
Tel. Code Each Per 10

PLATANUS ORIENTALIS Oriental Plane

8-10 ft., $11 / 4$ in. glance ... $\$ \$ 1.75 \quad \$ 16.00$

$10-11 \mathrm{ft} ., 1 \frac{1}{2}$ in. glancing $\quad 2.25 \quad 20.00$

$11-12 \mathrm{ft} ., 13 / 4$ in. glanced ... $\quad 2.75 \quad 25.00$

12-13 ft., 2 in. gland ... $\quad 3.00 \quad 27.50$

12-13 ft., $21 / 4$ in. glanders . . $\quad 3.25 \quad 32.50$

$12-14 \mathrm{ft} ., 21 / 2$ in. glandular . $\quad 5.00 \quad 47.50$

14-16 ft., 3 in. glandulous $\quad 7.50 \quad 70.00$

14-16 ft., 31/2 in. glare .... $10.00 \quad 80.00$

POPULUS BOLLEANA, S-10 ft.......glassily . . $1.50 \quad 12.00$

Pyramidal, Poplar

- BALSAMIFERA.

$8-10 \mathrm{ft} ., 1 \frac{1}{4}$ in. glazen . . . $\quad 1.00 \quad 8.50$

Balsam Poplar

8-10 ft., $11 / 2$ in. glazened . . $\quad 1.25$

10.00

$10-12 \mathrm{ft} ., 1^{3} / 4$ in. glazier . . $\quad 2.00 \quad 18.00$

- FASTIGIATA

10-12 ft., 2 n. glazing ... 2.50

Lommbardy Poplak

8-10 ft ........ gleamed . . .75

6.50

Low branched and

$10-12 \mathrm{ft} ., 1 \frac{1}{4} \mathrm{in} \mathrm{.} \mathrm{gleaming} \mathrm{.} 1.00$

8.50

straight leaders

11-13 ft., $1 \frac{1}{2}$ in. glean . . . . $1.50 \quad 12.50$

$12-14 \mathrm{ft} ., 13 / 1$ in. gleaned ... $2.00 \quad 17.50$

$\begin{array}{llll}13-15 \mathrm{ft} ., 2 & \text { in. gleaner ... } & 2.50 & \mathbf{2 2 . 5 0}\end{array}$

$14-16 \mathrm{ft} ., 21 / 4$ in . gleaning . $\quad 3.00 \quad 2750$

- MONILIFERA

8-10 ft., $11 / 4$ in. glen ..... $\quad .75$

6.50

Carolina Poplar

$11-12 \mathrm{ft} ., 1 \frac{1}{2}$ in. .glib . . . . 1.00

$12-13 \mathrm{ft} ., 1^{3} / 4$ in . gibly .... $1.50 \quad 12.50$

13-14 ft., 2 in. glibly ... $2.09 \quad 17.50$

$14-15 \mathrm{ft} ., 21 / 4$ in. glide .... $2.50 \quad 22.50$

$14-15 \mathrm{ft} ., 2^{1} / 2$ in . glider ... $\quad 3.50 \quad 32.50$

14-16 ft., 3 in. gliding ... $4.50 \quad 42.50$

PRUNUS (See Shrubs)

PTELIA TRIFOLIATA

$14-16 \mathrm{ft} ., 3 \frac{1}{2}$ in. . glided ... 5.00

Hop TrEe

4- $5 \mathrm{ft} \ldots \ldots$.... glistening .

.75

6.50

HOP TREE

6- $8 \mathrm{ft} \ldots \ldots \ldots$ gloamer . .

$6-8 \mathrm{ft} \ldots . .$. gloam .... 2.50

0.00

- BICOLOR

8-10 ft., $1 \frac{1}{4}$ in. .gloaming .

3.00

$10-12 \mathrm{ft} ., 11 / 2$ in. . gloat .... 3.50

$10-12 \mathrm{ft} ., 13 / 4$ in. gloated ... 4.50

- CERRIS, TURKEY OAK

$8 \mathrm{ft} ., 11 / 4$ in .... gloating... 2.25

$8-10 \mathrm{ft} ., 11 / 2$ in. globe . . . . 2.50

8-10 ft., $13 / 4$ in. .globose ... $\quad 3.00$

$8-10 \mathrm{ft} ., 11 / 4$ in . globing ... $\quad 2.50$

- COCCINEA

10-12 ft., $1 \frac{1}{2}$ in. glober ... 3.00

SCARLET OAK

6- $8 \mathrm{ft} ., 11 / 1$ in. glat ..... 2.00

Mossy Cup OAK

$8 \mathrm{ft} ., 1 \frac{1}{2}$ in.... glatty ... $\quad 2.50$

$8-10 \mathrm{ft} ., 13 / 4$ in. glast .... $\quad 3.00$

- MACROCARPA

Mossy Cup OAK

$10-12 \mathrm{ft} .11 \%$ in gloriously $\quad 3.00$

$10-12 \mathrm{ft} ., 2-2 \frac{1}{2}$ in glorify ... 4.00

$10-12 \mathrm{ft} ., 2 \frac{1}{2}-3$ in. glory .... 6.00

$12 \mathrm{ft} ., 3$ in....gloried ... 7.50

- PALUSTRIS

$8 \mathrm{ft} ., 11 / 4$ in ..... glorying . . 9.00

PIN OAK

$8-10 \mathrm{ft} ., 11 / 2$ in. gloss .... $\quad 2.50$

8-10 ft., $13 / 4$ in. glossily ... 3.50

10-12 ft., 2 in. glossiness . $\quad 4.50$

12-13 ft., 21/4 in. glossy ... 5.00

$12-13 \mathrm{ft} ., 21 \frac{2}{2}$ in. glove .... $\quad 6.00$

$12-1+\mathrm{ft} ., 3$ in. clowed. . . 7.50

12-14 ft., $31 / 2 \mathrm{in}$. glowing . 10.00

- PEDUNCULATA

8-10 ft........ glow..... 3.50

- PRINOS MICHAUXII

8-10 ft., 11/4 in . glummy . . 2.25

Chestnut OAK

$10-12 \mathrm{ft} ., 13 / 4$ in. .glutinous. .

3.50

$12-14 \mathrm{ft} ., 3 \frac{1}{2}$ in. . gluttony . . $\quad 6.00$

12-14 ft., 4 in. . . glutton ... 7.50

17.50

22.50

32.50

40.00

45.00

55.00

65.00

— ROBUR. PED. CONCORDIA

$4-5 \mathrm{ft} \ldots . . \ldots$ gnashed .. $\quad 3.50$

20.00

30.00

55.00

70.00 
QUERCUS-Continued
— RUBRA

RED OAK

RHUS COTINUS-Purple Mist OR FriNge (See Shrubs)

ROBINA PSEUDO ACACIA

SALISBURIA ADIANTIFOLIA GINKO

\section{SALIX BABYLONICA}

WeEPING WILLOW

- - AUREA PENDULA

- SOLOMONI

- CAPREA

- PENTANDRA

Laurel Leaf Willow

- ROSMARINIFOLIA, bush

- THURLOW, Elegantissima WeEping Willow

- VITELLINA AUREA GOLDEN BARK WILLOW - - BRITZENSIS

SOPHORA JAPONICA

Pagoda Tree

SORBUS AUCUPARIA-Evg. MT. Ash - - PENDULA, Weep. Mt. Ash .... TILIA AMERICANA

American Linden

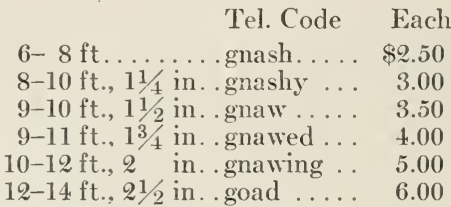

$6-8 \mathrm{ft} \ldots \ldots$.....goaded ... 1.00

$8-10 \mathrm{ft} \ldots . . .$. goading .. 1.50

$6-8 \mathrm{ft} \ldots . .$. goal ...... 1.50

$8-10 \mathrm{ft} ., 11 / 4$ in. goat .... $\quad 2.00$

$10-12 \mathrm{ft} ., 11 / 2 \mathrm{in}$. gobble ... $\quad 2.50$

10-12 ft., $13 / 4$ in. gobbled .. 3.00

10-12 ft., 2 in. gobbler ... 3.50

12-14 ft., $21 / 2$ in. goblin ... 4.00

$6-8 \mathrm{ft}$......... goblet . . .75

$8-10 \mathrm{ft} \ldots \ldots \ldots$ goddess .. . 1.25

$10-12 \mathrm{ft} \ldots \ldots \ldots$ godliness . 1.50

12-14 ft . . . . . godlike ... 2.00

$6-8 \mathrm{ft} \ldots . .$. godly ... $\quad 1.00$

8-10 ft. . . . . . godless ... 1.50

$6-8 \mathrm{ft} . . . \ldots$ godlessly . 1.25

$8-10 \mathrm{ft} \ldots \ldots \ldots$ godsend . . . . 1.50

$10-12 \mathrm{ft} \ldots . .$. . godson... 2.00

$12 \mathrm{ft} . . . . . .$. godspeed . $\quad 2.50$

$4-6 \mathrm{ft} \ldots \ldots \ldots$ going .... 1.00

6- $8 \mathrm{ft} \ldots \ldots$.......... 1.25

$4-6 \mathrm{ft} \ldots \ldots \ldots$ gold ..... .75

$6-8 \mathrm{ft} \ldots . .$. golden ... 1.00

8-10 ft........goldless .. 1.25

$2-3 \mathrm{ft} \ldots \ldots \ldots$ gone. . . . . . . . . . . .

$3-4 \mathrm{ft} \ldots . .$. . gong .... .75

$4-5 \mathrm{ft} \ldots \ldots$. gorge .... 1.00

6- $8 \mathrm{ft} \ldots \ldots \ldots$ gorgeous ... $\quad .75$

8-10 ft........ gorgeously $\quad 1.00$

$10-12 \mathrm{ft} \ldots \ldots \ldots$ gorging ... 1.50

4- $6 \mathrm{ft} \ldots \ldots$. . gosling... .75

6- $8 \mathrm{ft} \ldots . .$. . gospel .... 1.00

3- $4 \mathrm{ft} \ldots \ldots$. . . . gossiped .. .50

$4-5 \mathrm{ft} \ldots \ldots$. gossiping . .75

$5-6 \mathrm{ft} \ldots \ldots$. gossoon .. .85

$4 \mathrm{ft} \ldots . . . .2$. gothic ... . 2.00

$5 \mathrm{ft} \ldots \ldots \ldots$. . . . . . . 3.50

$6-8 \mathrm{ft} \ldots \ldots$. . . goutily ... 1.50

2 yr.heads ......governed 2.00

8- $9 \mathrm{ft} ., 11 / 4$ in . grab .... $\quad 2.00$

$9-10 \mathrm{ft} ., 11 / 2$ in . grabbed... $\quad 2.50$

$10-11 \mathrm{ft} ., 13 / 4$ in. grabber . . $\quad 2.75$

$11-12 \mathrm{ft}, 2$ in . grabbing . 3.00

$11-12 \mathrm{ft} ., 21 / 4$ in. grace .... 5.00

$12-13 \mathrm{ft} ., 2 \frac{1}{2} \mathrm{in}$. graced ... 6.00

$13-1+\mathrm{ft}, 3$ in graceful $\ldots \quad 7.00$

13-1 $4 \mathrm{ft}$., $3 \frac{1}{2}-4$ in. graceless . . 8.00

37.50

6.50

10.00

12.50

17.50

17.50

12.50

10.00

12.50

20.00

22.50

6.50

8.50

10.00

3.50

6.50

8.50

6.50

8.50

12.50

6.50

8.00

4.00

6.50

7.50

17.50

22.50

25.00

27.50

45.00

55.00

65.00

25.00

$8 \mathrm{ft} . .1 \frac{1}{4}$ in.... grade .... 3.00 $8-9 \mathrm{ft} ., 11 / 2$ in. . graded ... 3.25 9-10 ft., $13 / 4$ in. .grader ... $\quad 3.50$ 10-11 ft., $\mathcal{2}$ in. grading ... 4.50 $10-12 \mathrm{ft} ., 11 / 2 \mathrm{in}$. grafted ... 3.00 $12 \mathrm{ft}, 13 \frac{1}{4}$ in.... grafting .. 3.25 30.00 
TILIA-Continued

- - PLATYPHYLLA

Europ. Large Lvd. Linden

- - VULGARIS

European Linden

ULMUS AMERICANA

American Elm
Tel. Code

Each

Per 10

$8-9 \mathrm{ft}, 11 / 4$ in . grain .... 2.00

9-10 ft., $1 \frac{1}{2}$ in . grainer. . $\quad 2.50$

10-11 ft., $13 / 4$ in. .grained ... $\quad 3.00$

11-12 ft., 2 in. grammar $\cdot 3.50$

11-12 ft., $21 / 4$ in. . grandeur . $\quad 4.00$

$12-13 \mathrm{ft} ., 21 / 2$ in . grandly . . $\quad 5.00$

13-14 ft., 3 in. grange ... 6.00

$\$ 37.50$

47.50

55.00

13-14 ft., $3 \frac{1}{2}-4$ in.granite $\ldots \quad 7.50 \quad 70.00$

13-14 ft., $4-4 \frac{1}{2}$ in. grasper . . 10.00

8- $9 \mathrm{ft}, 1_{1 / 4}^{1 / 2}$ in . grasp ... $\quad 2.00$

9-10 ft., 11/2 in. . grasped . . 2.50

10-11 ft., $13 / 4$ in . grasping . . $\quad 3.00$

11-12 ft., 2 in. grass .... 3.50

11-12 ft., $21 / 4$ in. . grasslike . $\quad 4.00$

12-13 ft., 21/2 in. . grassy ... $\quad 5.00$

13-14 ft., 3 in. grated .... 6.00

13-14 ft., 31/2 in. .grateful . . 7.50

13-14 ft., 4 in. grate .... 10.00

$8-10 \mathrm{ft}, 11 / 4$ in gratify.. .1 .50$

9-11 ft., $1 \frac{1}{2}$ in . grating ... 2.00

$10-12 \mathrm{ft} ., 1^{3} / 4$ in. gratis ....

2.50

3.00

3.50

11-13 ft, $21 / 1$ in. gravely ...

$12-14 \mathrm{ft} ., 21 / 2$ in. . gravity ...

5.00

7.50

12-14 ft., 3 in. gravy.... .

12-14 ft., $3 \frac{1}{2}$ in. . graze .... .

8.50

3.50

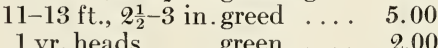

SCOTCH ELM

$1 \mathrm{yr}$. heads .....green .... 2.00

2 yr. heads ..... greet .... $\quad 2.50$

8-10 ft., $13 / 4$ in . greeting. . . 3.00

10-12 ft., 2 in. greeted ... $\quad 3.50$

WEEPING ELM

$6-8 \mathrm{ft}$. grief .... 2.00

- WELLESLEY

8-10 ft., $1 \frac{1}{4}$ in . grieving. .
18.00
$2.50 \quad 22.00$

\section{DECIDUOUS SHRUBS}

The shrubs we offer are of standard commercial grade, young and thrifty. They are not crowded in rows to make them grow tall but are given space enough for proper development This insures a bushy and full plant which far more than height ought to be, and, indeed is, the standard of excellence. Nearly all our shrubs are dug with a tree digger, thus insuring good roots, and are exactly what we represent them to be in all particulars.

\begin{tabular}{|c|c|c|c|}
\hline & Tel. Code & Each & Per \\
\hline ACER JAPONICUM AUREUM & $11 / 2-2 \mathrm{ft} \ldots \ldots$ pace & $\$ 2.00$ & \\
\hline Golden JaPan MaPle & $2-21 / 2 \mathrm{ft} \ldots \ldots \ldots$ pacer & 3.50 & \\
\hline & $3 \mathrm{ft} \ldots \ldots \ldots$ pacific $\ldots$ & 5.00 & \\
\hline - POLYMORPHUM & $2-3 \mathrm{ft} \ldots \ldots \ldots$ pacify & 2.50 & \\
\hline Green Leaved & .... packed & 3.00 & \\
\hline — ATROPURPUREUM & $.18-24$ in. & 2.50 & \\
\hline & $2-21 / 2 \mathrm{ft} \ldots$ & 3.50 & \\
\hline & $\begin{array}{l}21 / 2-3 \mathrm{ft} \ldots \ldots \ldots \text { packet } \\
3 \mathrm{ft} . \ldots \ldots \text { packing }\end{array}$ & $\begin{array}{l}4.50 \\
5.00\end{array}$ & \\
\hline - - DISSECTUM. & $11 / 2-2 \mathrm{ft}$ & 2.50 & \\
\hline Purple Cutleaf & ....ppadded & 3.50 & \\
\hline - - DISSECTUM ... & $2-21 / 2 \mathrm{ft} \ldots . . .$. paddled & 3.50 & \\
\hline $\begin{array}{l}\text { GREen Cut LEAF } \\
\text { - TARTARICA GIN }\end{array}$ & & & \\
\hline SibERIAN MAPLE & $4-5 \mathrm{ft}$. & 1.00 & $\$ 9.00$ \\
\hline AMELANCHIER POTRYAPIUM. & $2-3 \mathrm{ft} \ldots \ldots \ldots$ paganism & .65 & 5.00 \\
\hline JUNEBERRY & $3-4 \mathrm{ft} \ldots \ldots \ldots$ paganized & .75 & 6.50 \\
\hline & .....pagan & 1.00 & \\
\hline CANADENSI & ......paganizing & .65 & 5.50 \\
\hline & ......ppaged & .75 & 6.50 \\
\hline
\end{tabular}


AMORPHA CANESCENS

Tel .Code

Each Per 10

\section{AMORPHA CANESCENS}

- FRUTICOSA

False Ixdigo

AMYGDALUS FLOWERING ALMOND

Pink and White $\ldots \ldots \ldots \ldots \ldots \ldots . .11 / 2-2 \mathrm{ft} \ldots \ldots$ pagoda $\ldots$.

ANDROMEDA ARBOREA (See oxydendron)

\begin{tabular}{|c|c|c|c|}
\hline & Tel .Code & Each & Per 10 \\
\hline $15-18$ in. & pagan ... & $\$ .50$ & $\$ 4.00$ \\
\hline $18-2+$ in & pages & .65 & 5.50 \\
\hline $2-3 \mathrm{ft}$. & page. & .50 & 4.00 \\
\hline $3-4 \mathrm{ft}$. & pageant & .65 & 5.50 \\
\hline $\begin{array}{l}11 / 2-2 \mathrm{f} \\
2-3 \mathrm{ft}\end{array}$ & $\begin{array}{l}\text { - pagoda } \\
\text { paid }\end{array}$ & $\begin{array}{l}.65 \\
.85\end{array}$ & $\begin{array}{l}6.00 \\
7.50\end{array}$ \\
\hline
\end{tabular}

- MARIANA STAGgER Brsh .........18 in

ARALIA MANDSCHURICA

18 in. . . . . . p pain . ...
$+-5 \mathrm{ft} \ldots \ldots \ldots$ painful $\ldots$
$5-6 \mathrm{ft} \ldots \ldots \ldots$ painfulness
$2-3 \mathrm{ft} \ldots \ldots \ldots$ painfully
$3-4 \mathrm{ft} \ldots \ldots \ldots$ paining $\ldots$
$2-3 \mathrm{ft} \ldots \ldots \ldots$ painted $\ldots$

- PENTAPHYLla

1.00

1.50

- SPINOSA

Herceles' Club

3- $4 \mathrm{ft} \ldots \ldots$. . . painter ..

.50

.65

.50

t- $6 \mathrm{ft} \ldots \ldots \ldots$ painting . . $\quad 1.00$

$6-8 \mathrm{ft} \ldots \ldots \ldots$ paint ..... 1.25

8-10 ft. . . . . . . palace ... 1.50

ARONIA ARBUTIFOLIA NIGRA

BlaCk ChOKeberRy

$2-3 \mathrm{ft} . . . \ldots \ldots$ palate ....

$3-4 \mathrm{ft} \ldots \ldots$.... palatial ...

t- $5 \mathrm{ft} . . . \ldots \ldots$ pale ......

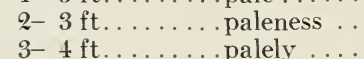

.65

.15

- RUBRA

$\begin{array}{rr}3-4 \mathrm{ft} \ldots \ldots \ldots \text { palely } \ldots . .7 & .75 \\ 4-5 \mathrm{ft} \ldots \ldots & 1.00\end{array}$

Red Chokeberry

AZALEA ARBORESCENS

Fragrant Azalea

$11 / 2-2 \mathrm{ft} . . \ldots \ldots$ palliate ...

$2 \mathrm{ft} \ldots \ldots \ldots \ldots$ palliated .

1.50

2.00

2.50

- CAlendulacea

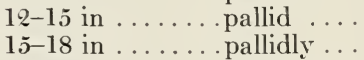

1.00

1.50

$18-2+$ in . . . . . . . pallidness . $\quad 2.00$

$2-21 / 2 \mathrm{ft} \ldots \ldots \ldots$ palling $\ldots .2 .50$

- KAEMPFERI

$12-15$ in....... . palm ....

2.00

$15-18$ in ....... palmate... 2.25

- MOLLIS

$12-15$ in ........

1.50

Japanese Azalea

$15-18$ in ........ palming...

2.00 colors mixed

$18-2+$ in ....... palmistry .

2.50

2.50

1.25

- NUDIFlORA ............... PiNkster Flower

18 in $\ldots \ldots \ldots$ palmy .....

$\begin{array}{ll}18-24 \text { in . . . . . . palsy .... } & 1.50 \\ 15-18 \text { in . . . . pampered . } & 2.00\end{array}$

- VASEYI............

$18-24$ in .........

2.50

12-15 in ....... pampering

$15-18$ in ........ pan ......

1.00

Sifall White Azalea

1.75

2.00

$2-3 \mathrm{ft} \ldots \ldots \ldots$ pancake .. $\quad .50$

BACCHARIS HALIMIFOLIA GRouxdsel Bush

$3-4 \mathrm{ft} . . . \ldots$. . panch .... $\quad .75$

BERBERIS HACKADATA

$11 / 2-2 \mathrm{ft} . . . \ldots$. paneled ..

$2-21 / 2 \mathrm{ft} \ldots \ldots \ldots$ pang ......

.50

.60

$21 / 2-3 \mathrm{ft} \ldots \ldots$. . pangs.... . . 75

- SIEBOLDI

$11 / 2-2 \mathrm{ft} \ldots \ldots \ldots$ panicle ...

$2-21 / 2 \mathrm{ft} \ldots \ldots \ldots$ panic $\ldots . .$.
$3-+1 \mathrm{ft} \ldots \ldots$. . . panicled .

.50

.60

- THUNBERGII

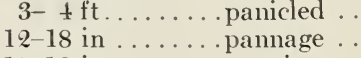

Thl一berg's Barberry

$15-18$ in ........

$11 / 2-2 \mathrm{ft}$. , extra. . panoply...

$2-21 / 2 \mathrm{ft} \ldots \ldots \ldots$ panorama

$21 / 2-3 \mathrm{ft} \ldots \ldots \ldots$ panoramic

- VULGARIS

$11 / 2-2 \mathrm{ft} \ldots \ldots \ldots$ panther ...

COMMOX BarberRY

$2-3 \mathrm{ft} \ldots \ldots$.... panting . .

- - PURPUREA

Purple Barberry

18-24 in ....... pantomine

$2 \mathrm{ft} \ldots \ldots \ldots \ldots$ pantry ....

$3 \mathrm{ft} . . . \ldots \ldots$. pant.....

2 yr........ papalize . .

BUDDLEIA VEITCHI

$11 / 2-2 \mathrm{ft} . . . \ldots$. paper ...

Perple Fruited

2-2 $1 / 2 \mathrm{ft} \ldots \ldots$. . papism ...

4.00

5.50

4.00

5.50

8.50

10.00

12.50

5.50

6.50

8.50

5.50

6.50

12.50

17.50

22.50

17.50

20.00

11.50

12.50

18.00

22.00

8.50

16.00

17.50

4.00

5.50

4.50

5.50

6.50 Per 100

$2.00 \$ 18.00$

25

.35

.45

.60

3.00

20.00

$4.00 \quad 25.00$

$5.50 \quad 35.00$

.75

7.00

45.00

.25

.40

.50

2.00

3.00

3.50

4.50

6.00

.40

.40

3.50

4.50 
CALYCANTHUS FLORIDUS

Strawberry SHRUb

CARAGANA ARBORESCENS Siberian Pea

\section{CARYOPTERIS}

- MASTACANTHA Blue Spirea

CEANOTHUS AMERICANA, RED ROot

\section{CELTIS OCCIDENTALIS} Nettle Tree

\section{CEPHALANTHUS}

OCCIDENTALIS

Burtton Bush

CERCIS CANADENSIS

American Judas

- JAPONICA JaPAN JUdAS

CHIONANTHUS VIRGINICA White Fringe

CLETHRA ALNIFOLIA Sweet Pepper

COLUTEA ARBORESCENS Bladder Senva

CORCHORUS (See Kerria).

CORNUS ALBA (SIBERICA) Red Branched

- ALTERNIFOLIA Alternate ELEGANTISSIMA

— SPAETHII, YelLOW

- KOUSA

Mascula Cornellan Cherry

- PAUCINERVIS

PANICULATA

Panicled Dogwood

- SERICEA

SILKY Dogwood

- STOLONIFERA

Red Osier

- - LUTEA

Golden BARK

CORYLUS AVELLANA.

- PURPUREA

Purple Filbert

COTONEASTER DIELSIANA
Tel. Code

$2-3 \mathrm{ft}$ papize ...

$3-4 \mathrm{ft}$

2- $3 \mathrm{ft} \ldots \ldots$. . . . paraclete

3- $4 \mathrm{ft}$....... parade

$4-5 \mathrm{ft} \ldots \ldots \ldots$ parading

15-18 in., 2 yr. . . paradise . .

18 in., 2 yr...... . paradox...

18-24 in ....... paradrome

$2 \mathrm{ft} . \ldots \ldots \ldots \ldots$......... paragon... $\quad .75$

3- $4 \mathrm{ft} \ldots \ldots \ldots$. . paragraph $\quad .50$

$4-6 \mathrm{ft} \ldots \ldots \ldots$ paragraphed .75

$6-8 \mathrm{ft} \ldots \ldots \ldots$ paragraphings 1.00

2- $3 \mathrm{ft} \ldots \ldots$.... parallel ... $\quad .40$

$3-4 \mathrm{ft} . . . \ldots \ldots$ paralleled . .50

$2-3 \mathrm{ft} \ldots \ldots \ldots$ paralysis . . $\quad .60$

$3-4 \mathrm{ft} \ldots \ldots \ldots$. . paralyzed . $\quad .75$

$4-5 \mathrm{ft} . . . \ldots \ldots$. paralyzing

$5-6 \mathrm{ft} \ldots \ldots \ldots$. . paralize

$11 / 2-2 \mathrm{ft} \ldots \ldots \ldots$ paramour .

2- $3 \mathrm{ft} \ldots \ldots$.... paraquet

3- $4 \mathrm{ft} . . . . .$. parasite ..

$4-5 \mathrm{ft} . . . \ldots \ldots$ parasol ...

$11 / 2-2 \mathrm{ft} \ldots \ldots$. parcel ...

2-21/2 ft....... parcenary

$21 / 2^{-3} \mathrm{ft} \ldots \ldots \ldots$ parched .

$3-4 \mathrm{ft} . . . \ldots \ldots$. parch ...

$2-3 \mathrm{ft} \ldots \ldots \ldots$ parching .

1.00

1.50

.75

1.50

1.75

2.50

.40

.45

.50

.75

.50

2- $3 \mathrm{ft} \ldots \ldots$....pardon

3- $4 \mathrm{ft} . . . \ldots \ldots$. pardonable

4- $5 \mathrm{ft} \ldots \ldots \ldots$ pardoned

2- $3 \mathrm{ft} \ldots \ldots \ldots$ pardoning

3- $4 \mathrm{ft} . . . \ldots \ldots$. paregoric.

$11 / 2-2 \mathrm{ft} \ldots \ldots$. . parentally

2-21/2 ft....... parenthesis

$3 \mathrm{ft} . . . \ldots \ldots$. parental .

$2-3 \mathrm{ft} \ldots \ldots \ldots$ parent ...

3- $4 \mathrm{ft} \ldots \ldots \ldots$. . . parentless

3- $4 \mathrm{ft} \ldots . . \ldots$. pargeted .

4- $5 \mathrm{ft} \ldots . . \ldots$... pargeting .

3- $4 \mathrm{ft} \ldots . . \ldots$. . parish ....

$2-3 \mathrm{ft} \ldots \ldots \ldots$..... paring ....

3- $4 \mathrm{ft} . . . . .$. park.....

$2-3 \mathrm{ft} . \ldots \ldots$.... parked...

3- $4 \mathrm{ft} . . . \ldots$... parker ...

$4-5 \mathrm{ft} \ldots \ldots \ldots$ parliament

2- $3 \mathrm{ft} \ldots \ldots$... parley ....

$3-4 \mathrm{ft} . . . \ldots \ldots$ parlor ....

4- $5 \mathrm{ft} . . . \ldots \ldots$ paroxysm .

2- $3 \mathrm{ft} \ldots \ldots \ldots$ parody ...

$3-4 \mathrm{ft} . . . . . .$. parole ...

4- $5 \mathrm{ft} . . . \ldots \ldots$. paroled ...

2- $3 \mathrm{ft} \ldots \ldots \ldots$. parquetry

3- $4 \mathrm{ft}$........ parquet .

4- $5 \mathrm{ft} \ldots \ldots \ldots$.... parried ...

$2-3 \mathrm{ft} . . . \ldots \ldots$.... parricide .

3- $4 \mathrm{ft} . . . \ldots$. . parr .....

$11 / 2-2 \mathrm{ft} \ldots \ldots$. parrot ...

2-2 $1 / 2 \mathrm{ft} \ldots \ldots$. parry ...

3-4 ft......... parse .....

$3 \mathrm{ft} . \ldots \ldots \ldots$........ parsley ...
Per 10

$\$ 4.50$

6.50

3.50

4.50

6.50

3.50

4.50

4.50

6.00

3.50

4.50

5.50

6.00

9.00

12.00

12.00

16.00

22.00

3.50

4.00

4.50

6.50

3.50

4.50

6.50

3.50

4.50

4.00

4.50

5.50

6.50

12.00

16.00

5.00

4.50

6.50

3.50

4.50

6.50

3.50

4.50

6.50

3.50

4.50

6.50

3.50

4.50

6.50

3.50

4.50

6.50

10.00

9.00 
CRATAEGUS (See Trees).

CYDONIA JAPONICA JAPAN QLTACE

- ALBA

White JAPAN QLTyCE

- MAULEI

DESMODIUM

- PENDULIFLORUM

DEUTZIA CANDIDISSIMA

Double White

- CRENATA, Fl. pl.

Double Pink

- GRACILIS

Slender Deutzla

- LEMOINEI

Lemoine's Deutzia

- PARVIFLORA

- PRIDE OF ROCHESTER

Large Flowered

- VILMORIN

DIERVILLA (See Weigela).

- TRIFIDA

ELEAGNUS ANGUSTIFOLIA

Russian Olive

- LONGIPES

GouMI

EUONYMUS ALATUS

JAPAN CORK BARKED

- AMERICANA

STRAWBERRY BCSH

- BUNGOIDES

- EUROPAEA

English Strawberry Tree

EXOCHORDA

- GRANDIFLORA

Pearl Besh

FORSYTHIA FORTUNEI

- INTERMEDIA

GoldeN Bell

- SUSPENSA

Fortune's Golden Bell
Tel. Code Each Per 10

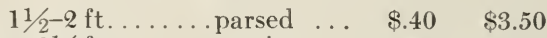

2-21/2 ft...... parsing ... $.45 \quad 4.00$

$21 / 2-3 \mathrm{ft} \ldots \ldots$. . partly ... $\quad .50 \quad 4.50$

$11 / 2-$ ift......part .... .50

2-21/2 ft ...... partable . $\quad .75$

$2 \mathrm{ft} \ldots \ldots \ldots$ partake $\ldots \quad 65 \quad 6.00$

$2-3 \mathrm{ft} \ldots \ldots \ldots$ party ... $\quad .50 \quad 4.50$

$3 \mathrm{ft} \ldots \ldots \ldots$ parts ..... $\quad .65 \quad 5.00$

$2-3 \mathrm{ft} \ldots \ldots . .$. participle . $\quad .50 \quad 4.50$

$2-3 \mathrm{ft} \ldots \ldots \ldots$ parting ... $\quad .40 \quad 3.50$

$3-4 \mathrm{ft} \ldots \ldots \ldots$. . partisan .. $.50 \quad 4.50$

$4-5 \mathrm{ft} . \ldots \ldots$.... partition . $\quad .75 \quad 6.50$

$2-3 \mathrm{ft} \ldots \ldots$..... partitive . $\quad .40 \quad 3.50$

$3-4 \mathrm{ft} \ldots \ldots \ldots$ partlet ... $\quad .50 \quad 4.50$

$4-5 \mathrm{ft} \ldots \ldots \ldots$ partly ....
.75

$12-15$ in ...... partook . . $\quad .40 \quad 3.50$

$15-18$ in . . . . . partridge. . $\quad .50 \quad 4.50$

18-24 in ....... partner ... $\quad .65 \quad 6.00$

$11 / 2-2 \mathrm{ft} \ldots \ldots$. . . passage ... $\quad .50 \quad 4.00$

$2-21 / 2 \mathrm{ft} \ldots \ldots$ passbook $\quad .65 \quad 6.00$

$21 / 2-3 \mathrm{ft} \ldots \ldots$ pass $\ldots . .75 \quad .75 \quad 7.00$

$11 / 2-2 \mathrm{ft} \ldots \ldots$ passable .. $\quad .40 \quad 3.50$

$2-21 / 2 \mathrm{ft} \ldots \ldots$. . passenger . $\quad .50 \quad 4.50$

$2-3 \mathrm{ft} \ldots \ldots \ldots$ passed $\ldots . \quad .40 \quad 3.50$

$3-4 \mathrm{ft} \ldots \ldots \ldots$ past ..... $\quad .50 \quad 4.50$

t- $5 \mathrm{ft} \ldots \ldots \ldots$.... passing ... $\quad .75 \quad 6.50$

$2-3 \mathrm{ft} \ldots \ldots \ldots$ passingly. . $\quad .50 \quad 4.50$

$\begin{array}{llll}3-4 \mathrm{ft} . \ldots \ldots \text { pasted } \ldots & .60 & 5.50\end{array}$

$18-24$ in ....... passion ... $\quad .40 \quad 3.50$

$2 \mathrm{ft} \ldots \ldots \ldots$ passible $\ldots . .50 \quad 4.50$

$4 \mathrm{ft} \ldots \ldots \ldots$ pass ..... $.75 \quad 6.50$

$2-3 \mathrm{ft} \ldots \ldots$....passionate. $\quad .40 \quad 3.50$

$3-4 \mathrm{ft}$........ . passioned . $\quad .50 \quad 4.50$

$4-5 \mathrm{ft} \ldots \ldots \ldots$ passivity . $\quad .75 \quad 6.50$

$2-3 \mathrm{ft} \ldots \ldots \ldots$ passport .. $\quad .50$

$3-4 \mathrm{ft} \ldots \ldots \ldots$ passively . $\quad .75$

18-24 in ...... past .... .50

$2-21 / 2 \mathrm{ft} \ldots \ldots$. . pasted ... $\quad .75$

4.50

7.00

$21 / 2-3 \mathrm{ft} \ldots \ldots \ldots$ pastel $\quad \ldots \quad 1.00 \quad 9.00$

$3-4 \mathrm{ft} \ldots \ldots$. . paste .... $1.25 \quad 11.00$

$2-3 \mathrm{ft} \ldots \ldots$.... pastoral... $\quad .40 \quad 3.50$

3- $4 \mathrm{ft}$........pastorate . $\quad .65 \quad 5.50$

$3-4 \mathrm{ft} . \ldots \ldots$.... pastorship. $\quad .50 \quad 4.50$

$\begin{array}{llll}4-5 \mathrm{ft} \ldots \ldots \ldots \text { pastor.... } & 75 & 6.50\end{array}$

$2-3 \mathrm{ft} \ldots \ldots \ldots$ pasture ... $\quad .50 \quad 4.50$

$\begin{array}{llll}3-4 \mathrm{ft} \ldots \ldots \ldots \text { pastured . . } & .75 & 6.50\end{array}$

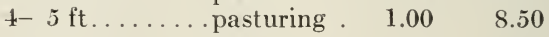

$18-24$ in . . . . . pastry ... . .50 4.00

$2-3 \mathrm{ft} \ldots \ldots \ldots$ pastry ... $\quad .60 \quad 5.00$

$3-4 \mathrm{ft} \ldots \ldots \ldots$ pat ...... $\quad .75 \quad 6.50$

$4 \mathrm{ft} \ldots \ldots . .$. patch ... $\quad 1.00 \quad 8.50$

$2-3 \mathrm{ft} \ldots \ldots \ldots$ patching . $\quad .40 \quad 3.50$

$3- \pm \mathrm{ft} \ldots \ldots \ldots$. . patchwork $\quad .50 \quad 4.50$

$\begin{array}{llll}4-5 \mathrm{ft} \ldots \ldots \ldots \text { patent } \ldots & .75 & 6.50\end{array}$

2- $3 \mathrm{ft} \ldots \ldots \ldots$ patermal . $\quad .40 \quad 3.50$

$3-4 \mathrm{ft} \ldots \ldots \ldots$...... paternity . $\quad .50 \quad 4.50$

$4-5 \mathrm{ft} \ldots \ldots \ldots$ path...... $\quad .75 \quad 6.50$

$2-3 \mathrm{ft} \ldots \ldots . .$. pathic .... $\quad .40 \quad 3.50$

$3-4 \mathrm{ft} \ldots \ldots$.... pathology $\quad .50 \quad 4.50$

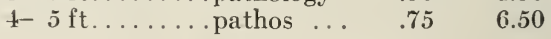


FORSYTHIA - Continued

- VIRIDISSIMA Dark Green Forsythia

FOTHERGILLAE

GENISTA SCOPARIA

Scotch Broom

- TINCTORIA

Dyer's Greenweed

HALESIA TETRAPTERA Silver Bell

HAMMAMELIS VIRGINICA WITCH HAZEL

HIBISCUS, AltheA, single . . Rose of Sharon

- Dbl. Pink, Purple, Red and White

- variegata

Variegated Leaved

Tree Form

HIPPOPHAEA SEA BuCKTHORN

HYDRANGEA ARBORESCENS. Wild Hydrangea

- - GRANDIFLORA ALBA Hills of SNOW

- NiVEA, Radiate

- OTAKSA

- Fiench Varieties, good assortment

- PANICULATA (Single Flowering)

\section{- - GRANDIFLORA}

Large Hydrangea

— - - standard (Tree Form)

QUERCIFOLIA, OAK LVD

\section{HYPERICUM AUREUM}

ST. JoHN's WorT

- DENSIFLORUM

- KALMIANUM

T ..............

. 2

- MOSERIANUM (GOLD Flower ....

ILEX SIEBOLDI

- VERTICILLATA (Prinos) ........ BLACK Alder

ITEA VIRGINICA

VIRGINIAN WILLOW

JASMINUM NUDIFLORUM

KERRIA JAPONICA Single Corchorus

- - FLOTA PLENA

- - VARIEGATA, VAR. $\mathrm{L}$.

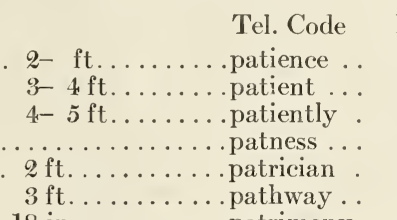

Fach Per 10

$\$ .40 \quad \$ 3.50$

$.50 \quad 4.50$

$.75 \quad 6.50$

.75

.50

.75

$\begin{array}{rr}18 \mathrm{in} \ldots \ldots \ldots \text { patrimony } & .40 \\ 2 \mathrm{ft} . \ldots \ldots \ldots\end{array}$

2- $3 \mathrm{ft} \ldots \ldots \ldots$ patriot...........

3- $4 \mathrm{ft} \ldots \ldots$. . patriotism. $\quad .95$

4- $6 \mathrm{ft} \ldots \ldots \ldots$. . patrol ... 1.50

2- $3 \mathrm{ft} \ldots \ldots$..... patron ... $\quad .50$

3- 4 it........patronage $\quad .75$

2- $3 \mathrm{ft} . . . \ldots .$. patroness . $\quad .40$

3- $4 \mathrm{ft} \ldots \ldots$..... patronize . $\quad .50$

Q $-3 \mathrm{ft} \ldots \ldots \ldots$..... patroon .. $\quad .40$

3-4 ft........patted ... .50

4- $5 \mathrm{ft} . \ldots \ldots$.... patter ... .65

$11 / 2-2 \mathrm{ft} . . . \ldots$. pattering. . $\quad .40$

$2-3 \mathrm{ft} \ldots \ldots \ldots$ pattern ... $\quad .50$

2- $3 \mathrm{ft} \ldots \ldots$.... patty ... $\quad .50$

$2-3 \mathrm{ft} \ldots \ldots \ldots$ pause.... $\quad .60$

3- $4 \mathrm{ft} . \ldots \ldots$...... pausing $\quad .75$

$18-24$ in . ...... pave .... $\quad .40$

2- $3 \mathrm{ft} \ldots \ldots \ldots$ paved .... .65

3- $4 \mathrm{ft} . \ldots \ldots$....pavement . $\quad .75$

2- $3 \mathrm{ft} \ldots \ldots \ldots$ paving ... $\quad .40$

$3-4 \mathrm{ft} . . . \ldots$. . pawing ... 50

4- $5 \mathrm{ft} \ldots \ldots \ldots$ pawed .... $\quad .75$

1 yr. ........ paw ..... .35

12-15 in ...... pawn ... $\quad .50$

15-18 in .......pawned .. .65

.65

.65

.75

.50

.65

.75

.50

.50

.65

.75

1.25

1.50

1.00

.40

.50

.40

.50

1.00

$3 \mathrm{ft} \ldots \ldots \ldots$ peaty $\ldots . .75$

$2 \mathrm{yr} . \ldots \ldots \ldots$ pebble $\ldots . .75$

$2-3 \mathrm{ft} \ldots \ldots \ldots$ peck..... 1.00

$11 / 2-2 \mathrm{ft} . \ldots \ldots$. . pecking .. $\quad .50$

$2-3 \mathrm{ft} \ldots \ldots$. . pectate ... $\quad .60$

$3 \mathrm{ft} \ldots \ldots \ldots \ldots$. . . . . . . $\quad .75$

18 in......... pectinate. . .40

2- $3 \mathrm{ft} \ldots \ldots \ldots$ pectinated $\quad 50$

.50

.50

.65

.50

.40

$15-18$ in .......
4.50

6.50

3.50

4.50

7.00

9.00

12.00

4.50

6.50

5.50

6.50

5.50

6.50

3.50

4.50

6.50

3.00

4.50

6.00

4.50

6.00

7.00

4.50

6.00

7.00

4.50

6.00

7.00

12.00

14.00

3.50

4.50

3.50

4.50

8.50

6.50

4.50

5.50

7.00

3.50

4.50

4.50

6.00

3.50 
LAURUS BENZOIN Sipir'E Bush

LESPEDEZA BICOLOR

Bush Clover

LIGUSTRUM AMURENSE AMOOR River

\section{- Ciliatum}

- IBOTA

\section{Tel. Code Each Per 10}

$11 / 2-2 \mathrm{ft} \ldots \ldots$. . peddled . $\$ .50 \quad \$ 1.00$

Q $-3 \mathrm{ft} \ldots \ldots \ldots$ peddler ... $.65 \quad 5.50$

$3 \mathrm{ft} \ldots \ldots \ldots \ldots$. . . peddling . . . .50

t $-\mathrm{t} \ldots \ldots \ldots \ldots$. . . pedestal .. $\quad .75$

$11 / 2-2 \mathrm{ft} . \ldots \ldots$. pedicle... $\quad 30$

$2-3 \mathrm{ft} \ldots \ldots \ldots$ pedigree .. . . 35

$3-4 \mathrm{ft} \ldots \ldots \ldots$. . . pediment . .50

$18-2+$ in . . . . . . peel ...... . . 40

$2-3 \mathrm{ft} \ldots \ldots \ldots$. peeling ... . .50

3.00

4.50

3.50

4.50

3.00

3.50

9-3 ft..............

$3-4 \mathrm{ft} \ldots \ldots \ldots$. peeping . . .50

4.50

$11 / 2-2 \mathrm{ft} \ldots \ldots$. . peerless ... $\quad .40$

2-21/2ft........ peerlessley $\quad .50$

- - REGELIANUM, true spreading Regel's Privet

\section{- LUCIDUM}

- OVALIFOLIUM, Califorsa

$1 \mathrm{yr} \ldots \ldots \ldots$...... peevishly . .50

well branched.

well branched.

12-18 in ......peg. .... . 10

well branched. .

$11 / 2-2 \mathrm{ft} \ldots \ldots \ldots$ pegged....

.15

well branched.

$2-3 \mathrm{ft} \ldots \ldots \ldots$ pegger $\ldots$

well branched, extra

$3-4 \mathrm{ft} \ldots \ldots \ldots$. pegging .

.25

.35

Globe Form

$5-6 \mathrm{ft} \ldots \ldots$. pellet ....

3.50

4.50

- STANDARD, 2 yr. head

18 in. diam. . . . . pence ... 1.50

1.50

$4-5 \mathrm{ft} \ldots \ldots$. . pelter.... 1.50

- AUREA GOLDEN PRIVET ..... 12-15 in ....... pelting...

- PULASKI ................. 2- $3 \mathrm{ft} \ldots \ldots \ldots$ pending ..

Polish Privet

- VULGARIS

$3-4 \mathrm{ft} \ldots \ldots \ldots$ pended ...

$2-3 \mathrm{ft} \ldots \ldots \ldots$ pend $\ldots \ldots$. . .
$3-4 \mathrm{ft} \ldots \ldots$ pendulum

Europeat

LONICERA ALBEKTI

$4-j \mathrm{ft} \ldots . .$. penetrant .

$1 \mathrm{ft} \ldots . . \ldots \ldots$ penetrate .

$11 / 2-\mathrm{ft} \ldots \ldots \ldots$. . . penetrated

q- $3 \mathrm{ft} \ldots \ldots \ldots$. . . . pencil ....

$3-4 \mathrm{ft} \ldots \ldots \ldots$ penetrating

$4-5 \mathrm{ft} . . . \ldots$ pent .....

2- $3 \mathrm{ft} \ldots . . .$. penitence .

3- $4 \mathrm{ft} \ldots \ldots$. . . penitency

2- $3 \mathrm{ft} \ldots \ldots \ldots$ penitential

$3-4 \mathrm{ft} \ldots . . \ldots$ pens ....

$2-3 \mathrm{ft} \ldots \ldots$... penitently

$3-4 \mathrm{ft} \ldots \ldots \ldots$. . . . penitent .

$4-5 \mathrm{ft} . . . \ldots$. . penit ....

2- $3 \mathrm{ft} \ldots \ldots$.... penman ..

$3-4 \mathrm{ft} . . . \ldots$. pennate ..

2- $3 \mathrm{ft} \ldots \ldots \ldots$. . . pemmating

$3-4 \mathrm{ft} \ldots \ldots$. . penned ...

$4 \mathrm{ft} \ldots \ldots \ldots$ penny ...

$2-3 \mathrm{ft} \ldots \ldots \ldots$ penniless .
$3-4 \mathrm{ft} \ldots \ldots \ldots$ penning .

2- $3 \mathrm{ft} \ldots \ldots \ldots$ pensioning

$3-4 \mathrm{ft} \ldots \ldots \ldots$ pensioned .

2- $3 \mathrm{ft} \ldots \ldots \ldots$ pensive ...

$3-4 \mathrm{ft} \ldots \ldots \ldots$ pensively

$4-5 \mathrm{ft} \ldots \ldots \ldots$ pent .....

.50
.40

.50

.30

.40

.50

.50

.65

.50

.50

.75

.50

.65

.40

.50

.50

.75

1.00

.50

.75

.50

.75

1.00

.50

.75

.50

.75

Standish Honersuckle

.40

Bush Hoxeysuckle

(Pink and IVhite)

MYRICA CERIFERA

12-18 in ....... pentecost

BIY BERRY

$11 / 2-2 \mathrm{ft} \ldots \ldots$. . . penurious .

$2-3 \mathrm{ft} \ldots \ldots \ldots$ penury . .

$3-4 \mathrm{ft}$....... peopled . .

$18-24$ in ....... people ...

2-21 $/ 2 \mathrm{ft} \ldots \ldots$..... peopled ..

2-3 $\mathrm{ft}$........ pepper ...

$3-4 \mathrm{ft} \ldots \ldots \ldots$ peppermint

.50

.75

.40

.60

.75

1.00

.50

.75

.75

1.00

Sorrel Tree

2 yr......... . peppered .

1.25

PAVIA MACROSTACHIA

12-18 in ......

1.50

Dwarf Horsechestwut

$18-24$ in .......

1.75

Per 100

$.80 \$ \$ 6.00$

$1.00 \quad 8.00$

2.00 10.00

$3.00 \quad 15.00$

$4.50 \quad 40.00$

12.50

12.00

4.50

3.50

$+.50$

2.50

3.50

4.50

4.50

6.00

4.50

4.50

6.00

3.00

4.50

4.50

6.50

4.50

6.50

4.50

6.50

9.00

4.50

6.50

4.50

6.50

3.50

4.50

6.50

3.00

5.00

7.00

9.00

4.50

6.50

7.00

9.00 


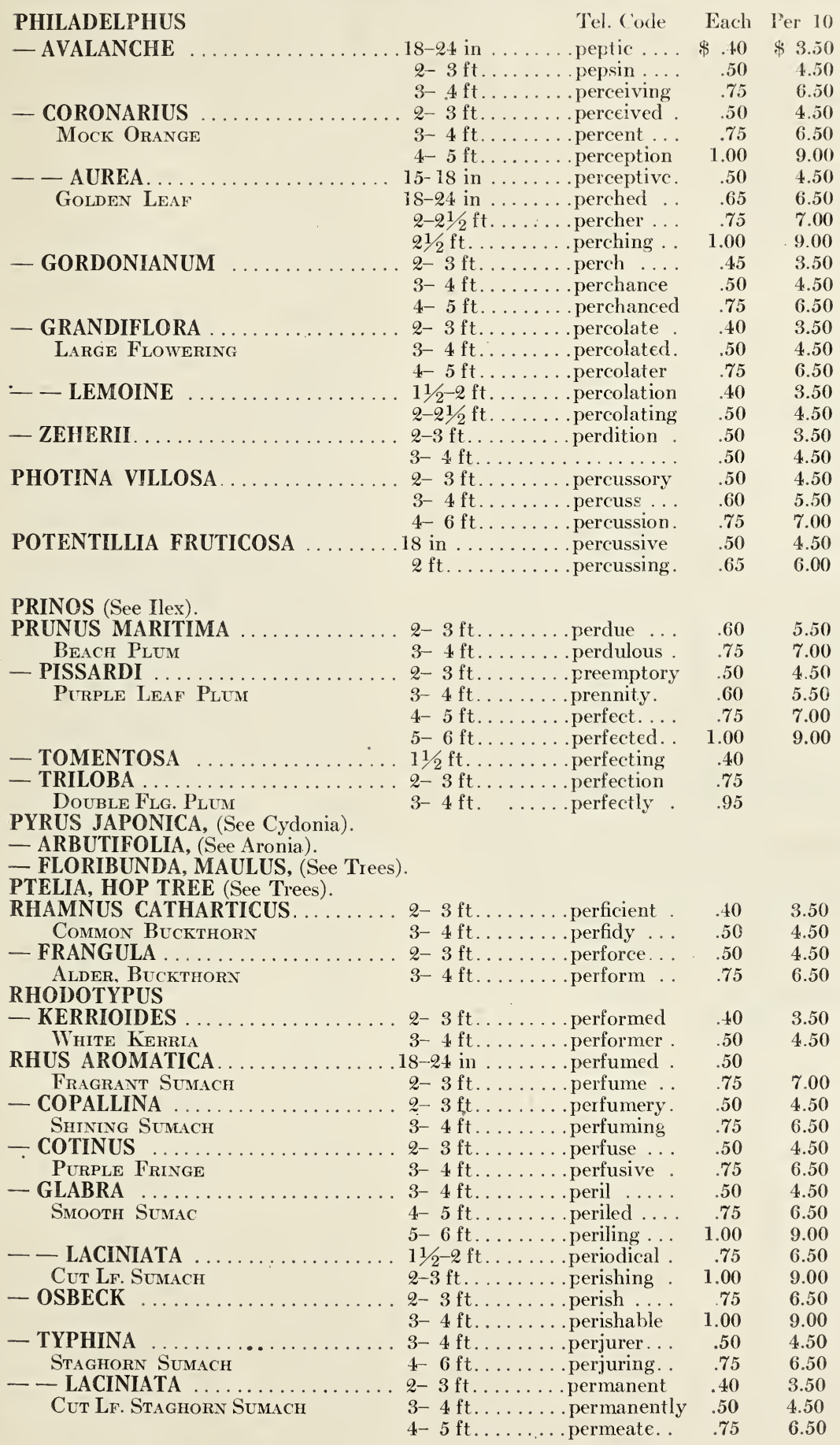


RIBES AUREUM

Yellow Flg. Ctrrant

- GORDONIANUM

PiNk Flg. Currant

- SANGUINEUM

Red Flg. Currant

ROBINA HISPIDA

Rose ACACIA

ROSA BLANDA, Meadow Rose

- CAROLINA

- LUCIDA, Glossy Rose

- MULTIFLORA JAPONICA

Japanese Climiding

- NITIDA

RUBIGINOSA, SweEt BRIAR

— RUBRIfOlia, (Ferreginea)

- Setigera, Pratrie Rose

- RUGOSA, RED

- ALBA, White

\section{- - HYBRIDS}

ROSA, CLIMBING AND TRAILING

- AMERICAN PILLAR ..........

- CRIMSON RAMBLER

- FLOWER OF FAIRFIELD

- PHILADELPHIA RAMBLER

- PINK RAMBLER

- WHITE RAMBLER

- YELLOW RAMBLER

- WICHURIANA

- - DR. VAN FLEET

- - DOROTHY PERKINS, PINK

- - DOROTHY PERKINS, White...

- - EXCELSA

- - GARDENIA.

- - HIAWATHA

- - LADY GAY

- HYBRID PERPETUAL, ETC

Good Assortment
Tel. Code Each Per 10

\begin{tabular}{|c|c|c|}
\hline $2-2 \mathrm{ft}$ & \pm 0 & \\
\hline$-3 \mathrm{ft} . . . . . .$. permissible & .50 & 4.5 \\
\hline$-3 \mathrm{ft} . . . \ldots \ldots$ permission & .40 & \\
\hline $3-4 \mathrm{ft} . \ldots \ldots \ldots$ permit & .50 & \\
\hline . . permitted & .75 & \\
\hline .....pernicious & .50 & \\
\hline $4 \mathrm{ft} \ldots \ldots \ldots$ pernicity & .65 & \\
\hline $3 \mathrm{ft} \ldots \ldots \ldots$ perpetrating & .50 & +.50 \\
\hline $4 \mathrm{ft} \ldots \ldots \ldots$ perpetrated & .75 & .5 \\
\hline . perpetual & .40 & \\
\hline . . perpetually & .50 & .5 \\
\hline . perpetuated & .40 & \\
\hline . perplexing. & .50 & \\
\hline ...perplex & .40 & \\
\hline ...perp & .50 & \\
\hline ......perp & .75 & \\
\hline $3 \mathrm{ft} \ldots \ldots \ldots$ per & .40 & \\
\hline $4 \mathrm{ft} \ldots \ldots \ldots$ perc & .65 & \\
\hline $2-2 \mathrm{ft}$ & .40 & \\
\hline $21 / 2 \mathrm{ft} \ldots \ldots \ldots$ pers & .50 & \\
\hline ......per & .40 & \\
\hline ...per & .50 & \\
\hline ...pers & .75 & \\
\hline rance & .40 & \\
\hline ...persevere & .50 & \\
\hline $4 \mathrm{ft} \ldots \ldots \ldots \mathrm{p}$ & .75 & \\
\hline . pers & .40 & \\
\hline .....pers & .50 & \\
\hline persisten & .50 & \\
\hline . persisting & .65 & \\
\hline personal. & .50 & \\
\hline ...personality & .65 & \\
\hline - personate & .75 & \\
\hline
\end{tabular}

$2-3 \mathrm{ft} \ldots \ldots . .$. persuade . $\quad .50$

4.50

$3-4 \mathrm{ft} . . . \ldots \ldots$. persuaded

2- $3 \mathrm{ft} \ldots \ldots \ldots$ persuading

$3-4 \mathrm{ft} \ldots \ldots \ldots$ persuasion

2- $3 \mathrm{ft} \ldots \ldots \ldots$ pert .......

$2-3 \mathrm{ft} . . . \ldots . .$. pertain ...

$3-4 \mathrm{ft}$........ pertaining

2- $3 \mathrm{ft} \ldots \ldots \ldots$ pertained .

$3-4 \mathrm{ft} . . . \ldots$. pertly ....

2- $3 \mathrm{ft} \ldots \ldots \ldots$ perusal ...

2- $3 \mathrm{ft} \ldots \ldots \ldots$ pervaded.

3- $4 \mathrm{ft}$......... Fervader .

2- $3 \mathrm{ft} \ldots \ldots \ldots$ pervading

3- $4 \mathrm{ft}$........ pervasion .

$2-3 \mathrm{ft} . . . \ldots \ldots$ perverse .

$3-4 \mathrm{ft} \ldots \ldots$. . . perversity

$2-4 \mathrm{ft} \ldots \ldots \ldots$ perverting.

3- $4 \mathrm{ft} \ldots \ldots \ldots$ pervert . .

2- $3 \mathrm{ft} \ldots \ldots \ldots$ pesting ...

$3-4 \mathrm{ft} \ldots \ldots$. . pested ...

2- $3 \mathrm{ft} \ldots \ldots \ldots$ pestilence

$3-4 \mathrm{ft} \ldots \ldots$. . . pet ....

2- $3 \mathrm{ft} . . . \ldots$. petition .

$3-4 \mathrm{ft}$......... . petitioner .

$2-3 \mathrm{ft} \ldots \ldots \ldots$..... petitioning

2- $3 \mathrm{ft} \ldots \ldots \ldots$ petrif ying

$3-4 \mathrm{ft} \ldots$. . . . petroleum

2- $3 \mathrm{ft} \ldots \ldots$.... pettish...

phaeton. . 
ROSA-Continued

Tel. Code Each Per 10

- - TEAS AND B

Good Assortment

- Tree Roses

phosphate $\$ 1.10$

$9: 50$

- Tree Rose.

picked

3.50

\section{LIST OF VARIETIES}

*HP indicates Hybrid Perpetuals; HT Hybrid Teas

American Beauty, HP, rose pink

Antoine Revoire, HT, rosy flesh.

Arthur R. Goodwin, HT, coppery rose.

Augustine Guinoisseau, HT, white.

Baby Rambler, red.

Baroness Rothchild, HP, pale rose.

Betty, HT, coppery rose.

Caroline Testout, HT, satiny rose.

Chateau de clos Vougeot, HT, maroon.

Dora Hansen, HT, peach pink.

Duchess of Wellington, HT, yellow.

Edward Mawley, HT, crimson.

Earl of Warwick, HT, salmon pink.

Etoile de France, H'T, crimson.

Etoile de Lyon, T, yellow.

Francis Scott, Key, H'T, red.

Frau Karl Druschki, HP, white.

Freidrichsruhe, HT, red.

General Jacqueminot, HP, red.

General MacArthur, H'T, scarlet.

General Superior Arnold Janssen, HT, carmine

Geo. C. Waud, HT, Vermillion.

Grus an Teplitz, HT, crimson.

Gustav Grunerwald, H'T, pink..

Hadley, H'T, crimson.

Hoosier Beauty, HT, crimson.

Hugh Dickson, HP, crimson.

Hugo Roller, T, yellow.

J. B. Clark, HT, scarlet.

Jonkheer, J. L., Mock, H'T, carmine.

Juliet, HT, old gold.

Kaiserine Augusta Victoria, HT, white.

Killarney, Pink, HT.

Killarney, White, HT.

Killarney, Queen, H'T, pink.

Killarney, Brilliant, HT, pink.

RUBUS ODORATUS. ..........

Flowering RASPBERRY

SAMBUCUS

- ACUTILOBA CT. LF.

- CANADENSIS

Common Elder

- NIGRA AUREA

GOLDEN ELDER

- RACEMOSUS

Red Berried Elder.............

SPIREA ARGUTA

- ATCHINSONI

BILLARDI

BUMALDI
Konigan Carola, HT, rose

Lady Alice Stanley, HT, rose.

Lady Hillingdon, HT, yellow.

Lady Ursula, HT, pink.

La France, HT, pink.

La Tosca, HT, pink.

Louise Catherine Breslau, HT, red.

Marquise de Ganay, HT, silver pink.

Maman Cochet, IT, pink.

May Miller, HT, coppery rose.

Miss Cynthia Forde, HT, rose pink.

Mme. Abel Chatenay, HT, golden pink.

Mme. Caroline Testout, HT, pink.

Mme. Edmee Metz, H'T, carmine.

Mme. Edouard Herriot, coppery yellow.

Mme. Jules Bouche, HT, white.

Mme. Jules Grolez, HT, salmon pink.

Mme. Ravary, HT, golden yellow.

Mme. Segond Weber, HT, rose salmon.

Mrs. Aaron Ward, HT, yellow.

Mrs. Arthur Robert Waddell, HT, pink

Mrs: John Laing, HP, pink.

Mrs. R. G. Sharman Crawford, HP, pink.

My Maryland, salmon pink.

Natalie Bottner, pale flesh.

Ophelia, HT, salmon pink.

Pharisaer, HT, rosy white.

Radiance, Red, HT.

Radiance, Pink, HT.

Reine Marie d'Italie.

Senatuer Mascuraud, HT, yellow.

Soliel d'Or, HT, golden yellow.

Ulrich Brunner, HP, cherry red.

Viscountess Folkestone, HT, creamy white.

Wm. R. Smith, H', soft pink.

Wm. Shean, H'T, pink.

\begin{tabular}{|c|c|c|}
\hline $2-3 \mathrm{ft} \ldots \ldots \ldots$ pickle & .50 & 4.50 \\
\hline $3-4 \mathrm{ft} \ldots \ldots$ pickled $\ldots$ & .65 & 6.00 \\
\hline $4-5 \mathrm{ft} \ldots \ldots \ldots$ pickling ... & .75 & 6.50 \\
\hline $3-4 \mathrm{ft} \ldots \ldots \ldots$ pillow & .40 & \\
\hline $2-3 \mathrm{ft} \ldots \ldots \ldots$ pillowed . . & .40 & 3.50 \\
\hline $3-4 \mathrm{ft} \ldots \ldots \ldots$ pillowing . & .50 & 4.50 \\
\hline $4-5 \mathrm{ft} \ldots \ldots \ldots$ pilot...... & .75 & 6.50 \\
\hline 2- $3 \mathrm{ft} \ldots \ldots \ldots$ piloting ... & .40 & 3.50 \\
\hline $3-4 \mathrm{ft} \ldots \ldots \ldots$ pilotage.. & .50 & 4.50 \\
\hline $4-5 \mathrm{ft} \ldots \ldots .$. piloted.... & .75 & 6.50 \\
\hline $2-3 \mathrm{ft} \ldots \ldots \ldots$ pincher . . . & .40 & \\
\hline $3-4 \mathrm{ft} \ldots \ldots \ldots$ pinched $\ldots$ & .50 & \\
\hline $4-5 \mathrm{ft} \ldots \ldots$ pinch $\ldots \ldots$ & .75 & \\
\hline-15 in. . . . . . pinching . & .35 & \\
\hline$-3 \mathrm{ft} \ldots \ldots \ldots$ pinion ..... & .50 & \\
\hline . . . pinioned . & .65 & 6.00 \\
\hline$-3 \mathrm{ft} \ldots \ldots \ldots$ pinking . . . & .40 & 3.50 \\
\hline$-4 \cdot f t \ldots \ldots$ pinked ... & .50 & 4.50 \\
\hline . . . pink & .75 & 6.50 \\
\hline aacle .. & .45 & 4.00 \\
\hline $21 / 2 \mathrm{ft} \ldots \ldots$. . pinnate ... & .50 & 4.50 \\
\hline
\end{tabular}


SPIREA - Continued

- - A. WATERER

Crimsox Spirea

\section{Tel. Code}

Each

Per 10

12-18 in ....... pinned ... \$ $\$ .5 \$ \$ 4.00$

$11 / 2-2 \mathrm{ft} \ldots \ldots$. . pinning ... $\quad .50$

$2-21 / 2 \mathrm{ft} \ldots \ldots$. pint . . . . . .60

4.50

5.50

$21 / 2 \mathrm{ft} \ldots \ldots \ldots$. . . . . . . . .

6.50

$\begin{array}{lll}12-18 \text { in . . . . . . piousness . } & .40 & 3.50 \\ 15-18 \text { in . . . . piously } & .5 & 4.00\end{array}$

DWARF WhITE SPIREA

$18-24$ in . . . . . pious .... . .60

5.50

$\begin{array}{lll}2-3 \mathrm{ft} \ldots \ldots \ldots \text { pioneer } \ldots & .40 & 3.50 \\ 3-4 \mathrm{ft} . & .50 & 4.50\end{array}$

3 rirs ........ piping ... $\quad .40 \quad 3.50$

- CRISPIFOLIA, (DWARF)

$2-3 \mathrm{ft} \ldots \ldots \ldots$ piper ......

3.50

4.50

7.50

3.50

4.50

6.50

- Goldex Nisebark

4- $5 \mathrm{ft} . \ldots \ldots \ldots$ piquantly .

2- $3 \mathrm{ft} \ldots \ldots \ldots$ piracy .... $\quad .40$

$3-4 \mathrm{ft} \ldots \ldots \ldots$ pirate ... . .50

$4-5 \mathrm{ft} \ldots \ldots$. pistol $\ldots . \quad .75$

- PRUNIFOLIA

Bridal Wreath

$11 / 2-2 \mathrm{ft} \ldots \ldots$. . pit ..... $\quad .40$

$2-3 \mathrm{ft} \ldots \ldots \ldots$ pitch .... . .50

$3-4 \mathrm{ft} \ldots \ldots \ldots$ pitcher ... $\quad .75$

- REVEESII FL. PL.

- ROTUNDIFOLIA

ROTYD Lid. SPIREA

- SALICIFOLIA

2- $3 \mathrm{ft} \ldots \ldots$.... piteous ... .5

3 - $+\mathrm{ft}$. . . . piteously -

4.50

6.50

4.50

6.50

2-3 ft.......... pitching .. $\quad .45 \quad 4.00$

$3 \mathrm{ft} \ldots \ldots \ldots$...... pitch .... .50 4.50

Q $-3 \mathrm{ft} \ldots \ldots \ldots$ pithy.... $\quad .40 \quad 3.50$

$3-4 \mathrm{ft} \ldots \ldots \ldots$. . . pith ..... $\quad .50 \quad 4.50$

- SORBIFOLIA

Q $2-3 \mathrm{ft} \ldots \ldots \ldots$ pitied..... $\quad .40$

Ash Lid. SPIRE.

$3-4 \mathrm{ft} \ldots \ldots \ldots$.... pitiful ... . .50

- THUNBERGII

$15-18$ in ....... pittance ..

.40

$18-24$ in . . . . . . . pitted ... . .45

Sxow Garlaid

\section{(1)}

- TOMENTOSA

$21 / 2-3 \mathrm{ft} \ldots \ldots \ldots$ pitying ...

.50

3.50

4.50

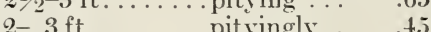

STEeple Bush

- VAN HOUTTEI

$3-4 \mathrm{ft} . . . \ldots$. . pivot .... . .50

4.00

4.50

Vax Holtte's Birdal Wreath

2- $3 \mathrm{ft} \ldots \ldots \ldots$ placed .... $\quad .40$

3.50

$3-4 \mathrm{ft} . . . \ldots$. . placid ... $\quad .50 \quad 4.50$

$4-5 \mathrm{ft} \ldots \ldots$. . placidly $\ldots \quad .75 \quad 6.50$

— VEITCHII

$2-3 \mathrm{ft} \ldots . . .$. placing

6.50
3.50

$3-4 \mathrm{ft} \ldots \ldots$. . placket $\ldots . \quad .50 \quad 4.50$

- WILSONI

$2-3 \mathrm{ft} . \ldots . \ldots$ plain .... . .50

STAPHYLEA COLCHICA, BLADDER NUT $2-21 / 2 \mathrm{ft} \ldots \ldots$. . plaintiff. . $\quad 1.50$

STEPHANANDRA FELXUOSA ...... $2-3 \mathrm{ft} \ldots \ldots \ldots$ plait..... .45

$3-4 \mathrm{ft} . . . \ldots \ldots$. . plan .... .50

t- $5 \mathrm{ft} \ldots \ldots \ldots$ planning . . . . . 5

STRAUNSVESIA

18 in..........planked .. .75

STYRAX JAPONICA

2- $3 \mathrm{ft} . \ldots \ldots$.... planking . .

$3-4 \mathrm{ft} . \ldots \ldots$....plant .... . . . 5

$4-5 \mathrm{ft} . \ldots \ldots$..... planted ... 1.00

\section{SYMPHORICARPOS}

RACEMOSUS $\ldots \ldots \ldots \ldots \ldots \ldots \ldots$. $1 \frac{1}{2}-2 \mathrm{ft} \ldots \ldots \ldots$ planting $\ldots . \quad .40$

WHITE SYOWBERRY

$11 / 2-2 \mathrm{ft} \ldots \ldots \ldots$ planting $\ldots$
$2-3 \mathrm{ft} . \ldots \ldots$ plaster $\ldots$

.50

Red INdLAN CuRraNT

$23 \mathrm{ft} \ldots \ldots \ldots$...... plattoon ..

.45

$3-4 \mathrm{ft}$....... platter ... .65

6.00

SYMPLOCOS CRATEGOIDES

18 in .......... playfully 1.50

SYRINGA JAPONICA

Japan Tree Lilac

2- $3 \mathrm{ft} . \ldots \ldots$.... playing ...

.50

$3-4 \mathrm{ft} . . . \ldots$.... player .... $\quad .75$

$4-5 \mathrm{ft} . \ldots . \ldots$. . playful ... 1.00

9.00

2-3 ft....... plea .... $\quad .50$

4.50

$3-4 \mathrm{ft} . . . \ldots$. plead .... $\quad .75$

6.50

$\begin{array}{rrr}4-5 \mathrm{ft}, \ldots \ldots \ldots \text {. . . pleading . } & 1.00 \quad 9.00\end{array}$

- VILLOSA, (Pink)

$2-3 \mathrm{ft} \ldots \ldots$..... pleaded .. .50

4.50

$3-4 \mathrm{ft} . . . \ldots$. . . pleader ... $\quad .75 \quad 6.50$

$4-5 \mathrm{ft} \ldots \ldots$. . pleasing... $\quad 1.00 \quad 9.00$ 
SYRINGA-Continued

- PERSICA. (Purple)

- - PRES. HAYES, (Purple)

- - ROTHOMAGENSIS, (Red)

- SANGEANA, (Red)

- ALBA, (IVhite)

- VULGARIS

Common Purple

- - ALBA

Common White

- ALPHONSE LAVELLE (Purple)

- CHAS. 10TH (Redish Purple)

- MICHAEL BUCHNER (Double Lilac)

- MARY LE GRAYE (Single White)

- MAD. LEMOINE. (Double White)

- MAD. CHAS. PERIER (Double White)

- - RUBRA DE MARLEY

(Light BLue)

- SCIPION COCHET

- SOUV DE L'SPAETH

(DARK Purple)

- WM. ROBINSON (Double PINK)

TAMARIX AFRICANA

- GALLICA

VACCINUM CORYMBOSUM

VIBURNUM ACERIFOLIA Maple Leaved

- CASSANOIDES

White RoD

- DENTATUM

Arrow Wó̀

- DILITATUM

- MOLLE

- LANTANA

WaYfaring Tree

- RUGOSUM

- LENTAGO

SheEpbuRY

- oXYCOCOS (American)

Highbush Cranberry

- OPULUS STERILIS

Common Snowball
Tel. Code Each Per 10

2-3 ft.......pleasant .. $\$ .50 \quad \$ 4.50$

$34 \mathrm{ft} \ldots \ldots \ldots$. . . please .... $\quad .75 \quad 6.50$

\&- $5 \mathrm{ft} \ldots \ldots \ldots$ pleased ... $1.00 \quad 9.00$

Q $-3 \mathrm{ft} \ldots \ldots$. . . pleasure .. $.50 \quad 4.50$

$3-4 \mathrm{ft} \ldots \ldots \ldots$. . . pledge .... $.75 \quad 6.50$

\& $3 \mathrm{ft} \ldots \ldots \ldots$. . . . . .50 4.50

$3-4 \mathrm{ft}$........plentiful . $\quad .75 \quad 6.50$

$4-5 \mathrm{ft} \ldots \ldots \ldots$. . . plenty . . . $1.00 \quad 9.00$

$2-3 \mathrm{ft} \ldots \ldots$.... plight ... $\quad .40 \quad 3.50$

$3-4 \mathrm{ft} \ldots \ldots \ldots$. . . plighted . . $\quad .65 \quad 6.00$

t- 5 ft....... plighting . $.85 \quad 7.50$

2- $3 \mathrm{ft} \ldots \ldots \ldots$. . . plodding . $\quad .50 \quad 4.50$

$3-4 \mathrm{ft} \ldots \ldots \ldots$. . . . . . . . . . . 65

2- $3 \mathrm{ft} \ldots \ldots \ldots$. . . . . . . . $50 \quad 4.50$

$3-4 \mathrm{ft}$....... plotted ... $\quad .75 \quad 6.50$

$4-5 \mathrm{ft} . \ldots \ldots$. plot ..... $1.00 \quad 9.00$

$2-3 \mathrm{ft} \ldots \ldots$...plough ... $\quad .50 \quad 4.50$

$3-4 \mathrm{ft} \ldots \ldots \ldots$. . . ploughing . $\quad .75 \quad 6.50$

2- $3 \mathrm{ft} \ldots . .$. plucking .. $\quad .50$

2- $3 \mathrm{ft} \ldots \ldots$. . plumbing . .50

$3-4 \mathrm{ft} \ldots \ldots$.... plume ... .75

2- $3 \mathrm{ft} \ldots \ldots \ldots$. . . . . . . . . . .

$3-4 \mathrm{ft} \ldots \ldots$....plunder .. $\quad .75$

2-3 ft........ plying ... . .50

3- $4 \mathrm{ft} \ldots \ldots \ldots$ poach .... .75

$4-5 \mathrm{ft} \ldots . .$. poaching . 1.00

3-4 ft. . . . . . poached . $\quad .75$

$11 / 2-2 \mathrm{ft} \ldots \ldots$ pocket ... .45

$2-3 \mathrm{ft} \ldots \ldots \ldots$ pod $\ldots \ldots . .65$

$2-3 \mathrm{ft} \ldots \ldots \ldots$ poem .... $\quad .60$

2- $3 \mathrm{ft} \ldots \ldots . .$. poetic ... .45

3-4 ft. . . . . . . poetry ... .50

$4-5 \mathrm{ft} \ldots \ldots \ldots$ pointed ... .75

$2-3 \mathrm{ft} \ldots \ldots \ldots$ point ..... .45

$3-4 \mathrm{ft} \ldots \ldots \ldots$ pointer ... .50

18 in. . . . . . . . .

$11 / 2-2 \mathrm{ft} \ldots \ldots$. . . . . .

2-3 ft....... . poke .... .50

$11 / 2-2 \mathrm{ft} \ldots \ldots$. poker .... .45

2-3 ft...... poking ... .55

$3-4 \mathrm{ft} \ldots \ldots$. . pole ..... .75

$2-3 \mathrm{ft} \ldots . . \ldots$ policy ... . .50

$3-4 \mathrm{ft} \ldots \ldots \ldots$ polite .... .65

$4-5 \mathrm{ft} \ldots \ldots \ldots$. . . politely ... .85

2- $3 \mathrm{ft} \ldots \ldots \ldots$ politeness . $\quad .50$

$3-4 \mathrm{ft} \ldots \ldots \ldots$. . . . political. . .75

2- $3 \mathrm{ft} \ldots \ldots$.... politics ... .50

3- $4 \mathrm{ft}$... . . . . polka ... . .65

$11 / 2-2 \mathrm{ft} \ldots \ldots \ldots$ polluting . . . . 45

$2-3 \mathrm{ft} \ldots \ldots \ldots$ pollution . $\quad .50$

$18-2+$ in . . . . . . . 45

2- $3 \mathrm{ft} \ldots \ldots \ldots$......

$3-4 \mathrm{ft}$....... popular . . $\quad .75$

2- $3 \mathrm{ft} \ldots . . \ldots$ poplar .... .50

3- $4 \mathrm{ft} \ldots \ldots \ldots$ porcelain . . . . .

4.50

6.50

4.50

6.50

4.50

6.50

9.00

6.50

4.00

5. 50

4.00

4.50

4.00

4.50

6.50

4.50

4.00

5.00

6.50

4.50

6.00

7.50

4.50

6.50

4.50

6.00

4.00

- +.50

$4.0 \mathrm{C}$

4.50

6.50

4.50

6.50

9.00

4.00

4.50

6.50

4.00

5.00

6.50 
VIBURNUM-Continued

- - NANA

Dwarf Sxowball

- PRUNIFOLIUM

BLACK HawK

- RHYTIDOPHYLLUM

- SIEBOLDI, JAPONICUM

- TOMENTOSUM

Single Flg.

- PLICATUM

JAPAN SxOWBaLL

- - Standard (Tree Form)

VITEX AGNUS CASTUS Chaste Tree

WEIGELIA AMABILIS Rose Colored

- CANDIDA, (White)

\section{- EVA RATHKE}

Ren FloweriNG

\section{- FLORIBUNDA}

- LAVELLI Red Flowerivg

- LUTEA, DIERVILLA

SESSIFOLIA

High Bush Honeyseckle

- NANA VARIEGATA

Variegated

- ROSEA

Rose Colored

XANTHORHIZA APIFOLIA

Yellow Root

\begin{tabular}{|c|c|c|}
\hline Tel. Code & Each & Per \\
\hline portance. & $\$ .35$ & $\$ 3.00$ \\
\hline $8-10$ in . . . . . portal & .45 & 4.0 \\
\hline . . portent & .5 & \\
\hline$-3 \mathrm{ft} \ldots \ldots \ldots$ portended & .65 & 6.0 \\
\hline . portend & .75 & 6. \\
\hline $4-j \mathrm{ft} \ldots \ldots \ldots$ postman .. & 1.00 & \\
\hline & 2.00 & \\
\hline ...... posture ... & .65 & 6.0 \\
\hline $3-4 \mathrm{ft} \ldots \ldots \ldots \mathrm{p}$ & .75 & \\
\hline $4-5 \mathrm{ft} \ldots \ldots \ldots$ pot & 1.00 & .0 \\
\hline $2-3 \mathrm{ft}$. & .5 & \\
\hline $3-4 \mathrm{ft}$. & .75 & 6.5 \\
\hline $4-5 \mathrm{f}$ & 1.00 & 8.5 \\
\hline $11 / 2-2 \mathrm{ft}$. & .50 & \\
\hline$-3 \mathrm{ft} .$. & .6. & 6 \\
\hline$\ldots \ldots p$ & 7. & \\
\hline $4-5 \mathrm{ft} \ldots \ldots \ldots \mathrm{F}$ & 1.00 & \\
\hline & 1.50 & \\
\hline $2-3 \mathrm{ft} \ldots \ldots \ldots \mathrm{r}$ & .50 & 4 \\
\hline$\cdots$ & .4 & 4.0 \\
\hline ... & .0 & \\
\hline $2-3 \mathrm{ft}$. & .45 & 4.0 \\
\hline $3-4 \mathrm{f}$ & .6 & 6.0 \\
\hline e... & .7 & \\
\hline t... & .4 & 4.0 \\
\hline s.. & .5 & 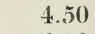 \\
\hline e $\ldots$ & .65 & 6.0 \\
\hline 1 & .75 & $.5 t$ \\
\hline$\cdots I$ & .45 & 0 \\
\hline$\cdots+1$ & .50 & .5( \\
\hline & .4 & 3.5 \\
\hline $3-4 \mathrm{ft}$. & .50 & 4.5 \\
\hline & & .5 \\
\hline & .5 & \\
\hline. $\mathrm{r}$ & .7 & \\
\hline & .4 & \\
\hline. $\mathrm{p}$ & .5 & \\
\hline$\ldots \mathrm{p}$ & .4 & 4.0 \\
\hline $\mathrm{p}$ & .5 & 0.0 \\
\hline$\ldots \mathrm{p}$ & .7 & 6.5 \\
\hline. $\mathrm{p}$ & .4 & \\
\hline$j-18$ in . . . . . princess . . & .50 & 4.5 \\
\hline
\end{tabular}

6.00

4.50

6.50

6.00

6.50

8.50

4.50

4.00

4.00

4.00

6.00

6.50

4.50

3.50

3.50

4.50

4.00

5.00

5.00

6.50

4.50

\section{CLIMBING VINES}

ACTINIDIA ARGUTA

Tel. Code Each Per 10 Silver Vine

$2-3 \mathrm{ft} \ldots \ldots \ldots$ ice $\ldots \ldots \ldots \$ .45$

AKEBIA, QUINATA.

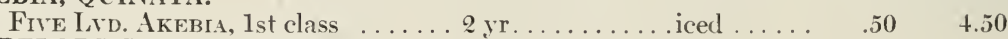

AMPELOPSIS ENGELMANI,

1 st class ................. 2 yr. . . . . . . icicle .... .45

— HETEROPHYLLA, VITIS . . . . . . 2 yr......... identify . . .50

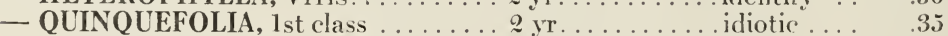

Tirginia Creeper

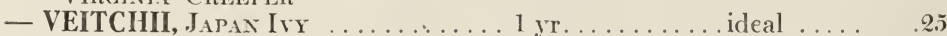

Field grown, medium. . . . . . . . . 2 gr . . . . . . . . .

Field grown, 1 st class. . . . . . . . . . Q 2 vr. . . . . . . . idly . . . . . .50

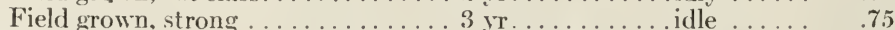


BIGNONIA RADICANS,

Tel. Code Each Per 10

Common Trumpet Vine........ $2 \mathrm{ft} \ldots \ldots \ldots \ldots$ ignore .... $.35 \quad 3.00$

- - GRANDIFLORA.

Chinese Trumpet Vine, $3 \mathrm{yr} \ldots \ldots \ldots \ldots \ldots \ldots$. $\ldots \ldots$ ignorance $\quad .75 \quad 6.50$

CELASTRUS SCANDENS $\ldots \ldots \ldots \ldots 2 \mathrm{y} \ldots \ldots \ldots \ldots$. . . . . . . . $\quad .50 \quad 4.50$

JAPAN STAFF VINE

CLEMATIS large flowering . . . . . . . . . . . . . . . illusion . . 1.00

— PANICUlata, Field Grown, extra $\ldots 2$ yr . . . . . . . . . . imagine $\ldots . \quad 40 \quad 3.50$

EUONYMUS (See Evergreens)

HEDERA HELIX .............. 3 in. pots . . . . implored. $\quad .45 \quad 4.00$

$\begin{array}{llll}4 \text { in. pots...... implore ... } & .50 & 4.50\end{array}$

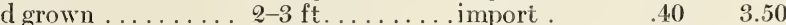

KUDZU VINE ................ 2 yr. . . . . . . impose. . . .55

LONICERA BRACHYPODA, JAPANESE 2 yr. . . . . . . . . . impress ... $\quad .50 \quad 4.50$

- AUREA, Japanese Golden . . . . 2 2 yr.......... impulsive . $\quad .45 \quad 4.00$

— HALLEANA, HaLL's J.PAN . . . . . . 2 yr. . . . . . . . inaugurate $\quad .45$

- HECKROTTI.

HeCKROT's Honeysuckle ....... 2 yr . . . . . . . . . incense . . $\quad .50 \quad 4.50$

— PUNICEA ..................... 2 yr ........... . .

LYCIUM BARBARUM ............. $2-3 \mathrm{ft} \ldots \ldots \ldots$ incline $\ldots . .45 \quad 4.45$

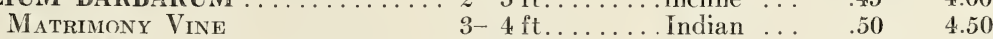

- SINENSIS $\ldots \ldots \ldots \ldots \ldots \ldots \ldots .2-3 \mathrm{ft} \ldots \ldots \ldots$ indicate $\ldots . .45 \quad 4.00$

$3-4 \mathrm{ft} \ldots \ldots \ldots$ index $\ldots . .50 \quad 4.50$

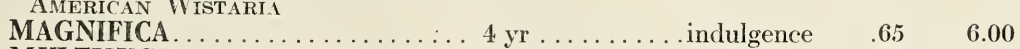

— MULTIJUGA ..... ........... $2-3 \mathrm{ft}$, heavy...infect .... $\quad .75 \quad 6.50$

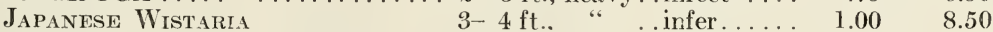

- SINENSIS $\ldots \ldots \ldots \ldots \ldots \ldots \ldots \ldots .3$ yr., " $\ldots$.inferior ... $\quad .75 \quad 6.50$

Chinese Wistaria 4 yr., “ . .infernal . $1.00 \quad 8.50$

- - ALBA ................. $2-3 \mathrm{ft} \ldots \ldots \ldots$ infirm .... .75

Chinese White $3-4 \mathrm{ft} \ldots \ldots \ldots$ infirmity 1.00

— standard, (Tree Form) . . . . . . . . . . . . . . . . . inform . . $\quad 3.50$

\section{CONIFERS AND EVERGREENS}

These have all been transplanted, most of them several times, giving ample room for full development, and frequently sheared to produce compact, symmetrical specimens. Those marked with a ${ }^{* *}$ will not be balled and burlapped at the prices named.

ABIES CILICICA, CicILIN Fir . . . . . . 18-24 in. . . . . . . sacredly . . \$2.25

Tel. Code Each Per 10

$2-21 / 2 \mathrm{ft} \ldots \ldots$. . . sacred .... 2.75

$18-24$ in ....... sacrificed. . 2.75

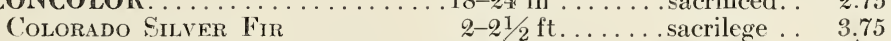

$21 / 2-3 \mathrm{ft} \ldots \ldots$. sad ...... 4.50

$3 \mathrm{ft} . .50$

- DOUGLASI .................18-24 in ......... saddened. . 1.50

$1.50 \quad \$ 12.50$

$2-21 / 2 \mathrm{ft} \ldots \ldots$.....saddening $\quad 2.00 \quad 17.50$

$21 / 2-3 \mathrm{ft} \ldots \ldots$..... saddle .... $2.50 \quad 22.50$

$3-4 \mathrm{ft} \ldots \ldots$. . . saddled ... $3.25 \quad 30.00$

$4-5 \mathrm{ft} \ldots \ldots \ldots$. . . . . . . $4.50 \quad 40.00$

- FRASERII.

$5-6 \mathrm{ft} \ldots \ldots$..... safer ..... 5.00

$2-21 / 2 \mathrm{ft} \ldots \ldots$. safely.... $\quad 2.50$

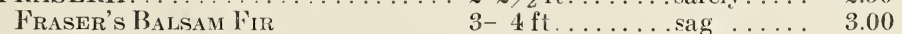

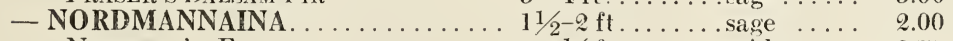

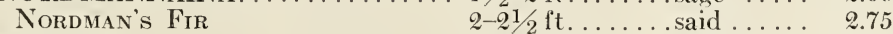

— OMORIKA …........... 3- 4 ft. . . . . . saint .... 4.50

— PECTINATA $\ldots \ldots \ldots \ldots \ldots \ldots \ldots .2-21 / 2 \mathrm{ft} \ldots \ldots \ldots$ salay $\ldots . .2 .50$ 
ABIES-Continued

- VEITCHII

VeITCH's Fir

White Sprece

- CANADENSIS

Black HiLL'S SPRTCE

- EXCELSA

NorwaY sprece
- PICEA ALBA

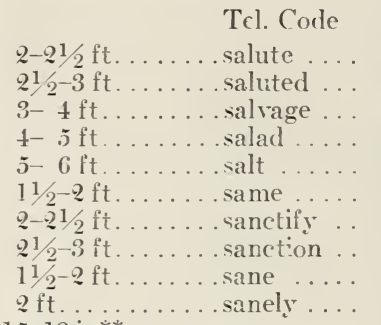

$15-1 \mathrm{~s}$ in $* * \ldots$ sap $\ldots . . . .$.

$11 / 2-2 \mathrm{ft} . \ldots \ldots$. . sardine ... 1.00

$2-21 / 2 \mathrm{ft} \ldots . .$. sardonic . 1.25

$21 / 2-3 \mathrm{ft} \ldots \ldots$. . . . . . . . . . 1.50

$3-4 \mathrm{ft} \ldots . .$. . sat . . . . . 2.25

$4-5 \mathrm{ft} \ldots . . .$. satan .... 3.50

$7-8 \mathrm{ft} \ldots \ldots . .$. satisf $\mathrm{y} \quad \ldots .6 .00$

18 in .......... saucer .... 200

AlREA, GoldeN SPRtCe.

$18 \mathrm{in} \mathrm{.........} \mathrm{sauce} \mathrm{...} 2.50$

$2 \mathrm{ft} \ldots \ldots \ldots$................ 2.75

12 in ..........sauntering 1.75

18 in .......... sauntered . $\quad 2.50$

Q-2 $1 / 2 \mathrm{ft} . \ldots$. . savage ... 2.7 .5

$3-4 \mathrm{ft} \ldots$...... savagely . 3.7 .5

12 in .......... saving ... 2.25

$\mathcal{Q}-\mathcal{Q} 1 / 2 \mathrm{ft} \ldots \ldots$. . . scalding... $\quad 2.50$

$3-4 \mathrm{ft} \ldots . .$. . . scallop ... 4.00

$1 \frac{1}{2} \mathrm{ft} \ldots \ldots . .$. sean ..... 1.75

$2 \mathrm{ft} \ldots \ldots \ldots$....... scandal ... $\quad 2.50$

$21 / 2 \mathrm{ft} \ldots \ldots \ldots$. . . scanned . . $\quad 2.75$

$3 \mathrm{ft} \ldots \ldots \ldots$........ scanning . $\quad 3.50$

$4 \mathrm{ft} \ldots . . . . . . . .5$ scant ..... 5.00

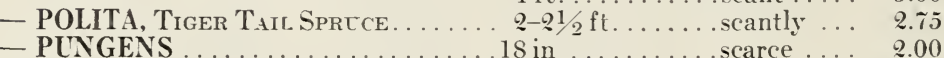

Qft............

$21 / 2 \mathrm{ft} \ldots \ldots$. . . scarceness 2.75

$3 \mathrm{ft} \ldots . . . \ldots$.... scare . ... 3.25

$4 \mathrm{ft} \ldots \ldots . . . .$. scared .... 5.00

$21 / 2 \mathrm{ft} \ldots \ldots$. . . scholar ... 6.50

$3 \mathrm{ft} \ldots \ldots . . . . . . . . .5$ scholarly $\quad 7.50$

$4 \mathrm{ft}$.............school .... 9.50

Q-21 $1 / \mathrm{ft} \ldots . .$. . schooner . $\quad 2.00$

$21 / 2-3 \mathrm{ft} \ldots \ldots \ldots$ scoff $\ldots \ldots, \quad 2.50$

18 in...........scoffed ... 1.75

$2-21 / 2 \mathrm{ft} \ldots \ldots \ldots$ scoffing ... $\quad 2.50$

ANDROMEDA CATESBAEI

(LEUCOTHOE)

- FLORIBUNDA

Mountain Fetter Bush

AZALEA AMOENA

12 in... . . . . . scolding. . .

12 in ............scorch ...

2.50

$12-15$ in .........scouring . . 2.50

15-18 in ......... scoured ... 2.75

$18-2 \frac{1}{2}$ in ........ scouted ... 3.00

$18-24$ in. ex.bushy scow ... 3.50

$12-15$ in . . . . . scowl ... 2.50

$15-18$ in ...... scowler ... 2.75

$18-24$ in ..........scowled .. 3.00

- INDICA ALBA

$12-15$ in . . . . . . scowling . $\quad 2.00$

18 in .......... scramble . 3.00

$11 / 2-2 \mathrm{ft} \ldots \ldots$. . . scraping . . .75

2-21/2 ft........scraper ... .9.5

$11 / 2-2 \mathrm{ft} \ldots \ldots \ldots$ scratching 1.25

2-2 $1 / 2 \mathrm{ft} \ldots \ldots$. . seratch ... 1.75

$21 / 2-3 \mathrm{ft} \ldots \ldots$. . scrawling . $\quad 2.50$

$3-4 \mathrm{ft} . . . . .$. scrawled . $\quad 3.00$

$4 \mathrm{ft} \ldots \ldots . .$. serawl .... 3.75
Per 10

$\$ 29.50$

30.00

4000

50.00

60.00

10.00

29.50

20.00

22.50

6.50

9.00

10.00

12.50

20.00

32.50

15.00

20.00

22.50

30.00

17.50

20.00

15.00

17.50

22.50

10.00

15.00 
BIOTA-Continued

Tel. Code Each Per 10

- - AUREA CONSPICUA, Golden . . 18 in ........... scrawler . . $\$ 2.00$

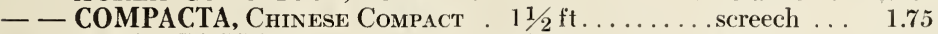

— ELEGANTISSIMA $\ldots \ldots \ldots \ldots .11 \frac{1}{2} \mathrm{ft} \ldots \ldots \ldots$ screw $\ldots . .1 .75$

Rollinson's Golden $2 \mathrm{ft} \ldots \ldots \ldots \ldots$.................. 2.75

$\$ 15.00$

$2.7 .5-25.00$

$21 / 2 \mathrm{ft} \ldots \ldots \ldots$..... scrubbing . $\quad 3.00 \quad 28.00$

$3 \mathrm{ft} \ldots \ldots \ldots$......... scrub $\ldots . .3 .75 \quad 35.00$

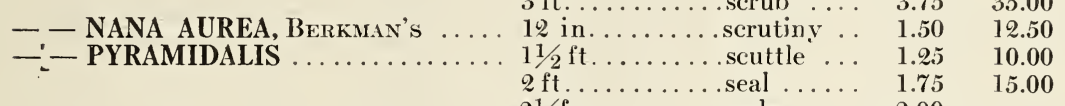

BUXUS ARBORESCENS

Tree Box (Bush Form)

$21 / 2 \mathrm{fr} \ldots \ldots$. . . sealer .... 2.00

$3 \mathrm{ft} . \ldots \ldots$............. 2.75

12 in ............... searching . 1.50

15 in . . . . . . . . searched . . $\quad 2.00$

$11 / 2 \mathrm{ft} \ldots \ldots \ldots$.............. $\quad 2.75$

Q-2 $1 / 2 \mathrm{ft} \ldots \ldots \ldots$. . . . . seasoned . . 5.00

$3-3 \frac{1}{2} \mathrm{ft} . . . \ldots$. . . seat ..... 9.00

2 in ................ .35

3 in ............. ..5ecure ... .55

$6-8$ in.** . . . . . sedately . . $\quad .35$

$10-12$ in ........ sedative .. .50

$2 \mathrm{ft} \ldots \ldots . . .$. select .... 2.00

$3 \mathrm{ft} \ldots \ldots \ldots \ldots$. . . . . . . . . 2.50

12-18 in . . . . . . selling .... 1.50

$11 / 2-2 \mathrm{ft} . \ldots \ldots$ self $\ldots . . .2 .00$

12 in . . . . . . . . semble ... 1.50

$4 \mathrm{ft}$.............senator ... 2.00

2-21 $1 / 2 \mathrm{ft} \ldots \ldots \ldots$ send ....... 2.50

$21 / 2-3 \mathrm{ft} \ldots \ldots$. . . sense . . . . 3.25

$3-4 \mathrm{ft} \ldots \ldots \ldots$...... senseless . 4.50

2 yr................

EUONYMUS ACUTUS

- JAPONICUS.

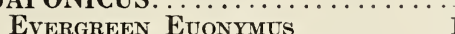

- PULCHALIUS

5-18 in ........ sentiment .

$18-24$ in ..........

12 in .............

- RADICANS, 2 yr. bushy $\ldots \ldots \ldots \ldots \ldots \ldots \ldots \ldots \ldots \ldots$ separate.

CARRIERI

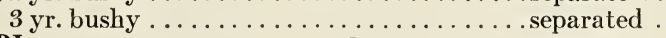

- CARRIERI..............................

- RADICANS VARIEGATA,

3 yr. bushy $\ldots \ldots \ldots \ldots \ldots \ldots \ldots \ldots \ldots \ldots$
-

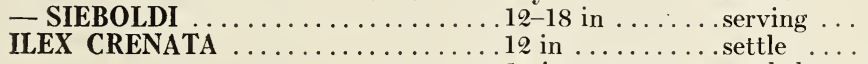

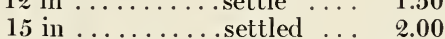

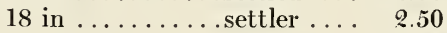

JUNIPERIUS CANADENSIS

15 in ........... severe ... 1.25

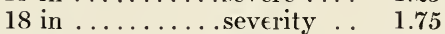

$2 \mathrm{ft} \ldots \ldots \ldots \ldots$ sewed $\ldots . . .2 .50$

- COMMUNIS-Common Juniper $\ldots 18$ in $\ldots \ldots \ldots \ldots$ sew $\ldots \ldots . \quad 2.00$

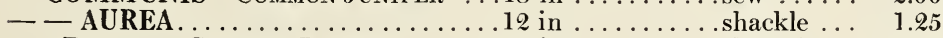

Douglas Golden Juniper 15 in $\ldots \ldots \ldots \ldots$ shaded.... 2.00

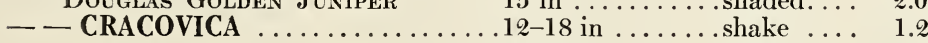

- - HIBERNICA

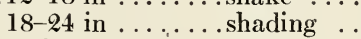

1.25

1.50

1.00

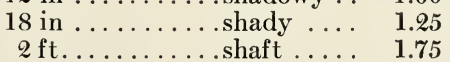

$\begin{array}{rr}2 \mathrm{ft} \ldots \ldots \ldots \ldots \text { shaft } \ldots \ldots & 1.75 \\ 21 / 2 \mathrm{ft} \ldots \ldots \ldots \text { shafting.... } & \mathbf{2 . 2 5}\end{array}$

$11 / 2 \mathrm{ft} \ldots \ldots \ldots$. . . . shaking .. $\quad \mathbf{2 . 7 5}$

- PROSTRATA

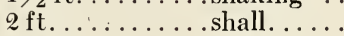

$21 / 2 \mathrm{ft} \ldots \ldots \ldots$. . . . shallow ...

$3 \mathrm{ft} \ldots \ldots \ldots$...................

$11 / 2 \mathrm{ft} \ldots \ldots \ldots$. . . . . . . . .

$2 \mathrm{ft} \ldots \ldots \ldots$........................

1.25

2.50

3.50

1.50

2.00

SWEDISH JUNIPER

.12 in ............

1.50

$11 / 2 \mathrm{ft} \ldots \ldots \ldots$. . . . . shamed ... $\quad 2.50$

- JAPONICA

12-18 in ..............

2.75

JAPAN JUNIPER

$18-24$ in $\ldots . .$. . shanty $\ldots .3 .50$
Per 100

200.00

$5.00 \quad 25.00$
10.00

17.50

10.00

12.50

9.00

10.00

15.00

25.00

12.50

17.50

12.50
4.00

5.00

3.00

.50 
JUNIPERIUS-Continued

- - GLOBOSA

Tel. Code Each

Per 10

- VARIEGATi

10 in. ${ }^{* *} \ldots \ldots$ sharpen .... $\$ 1.75$

JAPAN VAR. JUNIPER

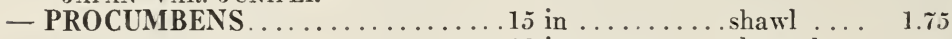

18 in ................ 2.50

- SABINA.

12-15 in ........ sheath ... 1.50

SAVIN JUNIPER

$15-18$ in .........sheathing . $\quad 2.00$

$11 / 2 \mathrm{ft} \ldots \ldots \ldots$ shed ...... 2.50

TAMARISCIFOLIA

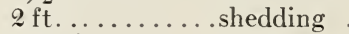

2.75

8-12 in .........sheltered . 1.75

SINENSIS

$3 \mathrm{ft} . . . . . .$. . shield.... 4.00

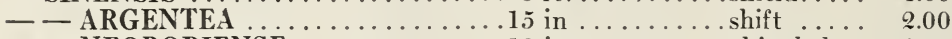

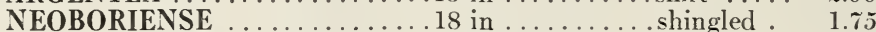

$2 \mathrm{ft} . \ldots \ldots \ldots$. . . . . . $\quad 2.50$

- - PFITZERIANA

12-18 ill .............. . . . . . . .

1.50

$11 / 2-2 \mathrm{ft} \ldots \ldots$. . . shirt. ..... 2.00

$2-21 / 2 \mathrm{ft} \ldots \ldots$. . shiver .... 2.75

3- $4 \mathrm{ft}$........ shivering . $\quad 5.00$

- VIRGINIANA

$11 / 2 \mathrm{ft} \ldots \ldots \ldots$ shortened . $\quad 1.00$

Red Cedar

$2 \mathrm{ft} \ldots \ldots \ldots$............. 1.25

$21 / 2 \mathrm{ft} \ldots \ldots \ldots$ should .... 1.75

$\$ 12.00$

15.00

20.00

12.00

18.00

25.00

9.50

10.00

Sheared plants

$3-4 \mathrm{ft} . \ldots \ldots$ shoulder ... 2.50

$4-5 \mathrm{ft} . \ldots \ldots$..... shout $\ldots . .4 .50$

$5 \mathrm{ft} . \ldots \ldots$................ shouted .. 50

$6 \mathrm{ft} . \ldots \ldots$........ shouting . . 6.50

- PENDULA

$3 \mathrm{ft} . \ldots \ldots$........ shrewdish . $\quad 3.75$

WeEPiNg Juniper $4 \mathrm{ft} \ldots \ldots \ldots \ldots$ shrewdness 4.50

- W.AUKEGAN

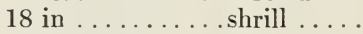

1.75

KALMIA LATIFOLIA (Nur. grown) . . 15-18 in . . . . . shrimp. . 1.75

Mountain Latrel $\quad 11 / 2-2 \mathrm{ft} \ldots \ldots$ shrine .... 2.50

$2-21 / 2 \mathrm{ft} \ldots \ldots$. . . shrink .... 2.75

$21 / 2 \mathrm{ft} \ldots \ldots$...... shrinkage . $\quad 3.50$

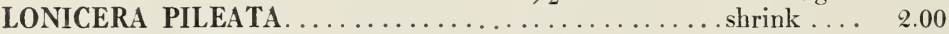

MAHONIA AQUIFOLIA ......... 12-15 in ......... shudder . . 90

Holl. Lvd. Ashbery . . $15-18$ in ...............

18 in ..................... 1.25

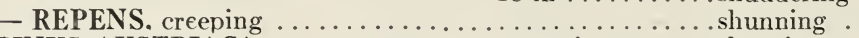

PINUS AUSTRIACA ........... $15-18$ in ............

Acstrian Pine $11 / 2-2 \mathrm{ft} \ldots \ldots$. $\ldots$ shun ..... 1.25

2-Q $1 / 2 \mathrm{ft} \ldots \ldots$................. 2.00

$21 / 2-3 \mathrm{ft} \ldots \ldots$. . . shyness ... 2.25

$3-4 \mathrm{ft} . \ldots . .$. sick ..... 3.00

t- $5 \mathrm{ft} \ldots . . .$. sickening. . 5.00

$5-6 \mathrm{ft} \ldots \ldots \ldots$ sickened .. $\quad 7.50$

$6-7 \mathrm{ft} \ldots \ldots$............ 10.00

- CEMBRA

12 in .......... sickle ... 1.75

Swiss Stone Pine

15 in ......... sickly.... 2.25

18 in ...........sickness .. 2.75

- DENSIFLORA

$2 \mathrm{ft} . . . \ldots \ldots$....... sifter .....

2.25

Japanese Red Pine

$21 / 2 \mathrm{ft} \ldots \ldots \ldots$.............

2.75

$3 \mathrm{ft} \ldots \ldots . . .$. sift ...... 3.00

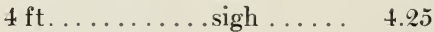

- EXCELSA

18-24 in ........ sightless . .

1.25

Bothay Pixe

2-21/2 ft. . . . . . sightly ...

$21 / 2-3 \mathrm{ft} \ldots . .$. sigh ......

1.75

2.50

$3 \mathrm{ft} \ldots . . . .$. sighed .... 3.50

- FLEXILIS

18 in ........... signature.

2.00

$2 \mathrm{ft} \ldots \ldots \ldots \ldots \operatorname{sign} \ldots \ldots . . . .50$

$21 / 2 \mathrm{ft} \ldots \ldots \ldots$. . . . . 3.25

- MUGHO

15-18 in ........ silence ...

Dwarf Mt. Pine

$18-24$ in ...........silent ...

2.25

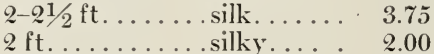

- MONTiCOLA Mt. White Pine

$2 \mathrm{ft} . \ldots \ldots \ldots \ldots$ sill . . . . . . 1.75

$21 / 2 \mathrm{ft} \ldots \ldots \ldots$...... silver .... 2.50

16.00

9.00

10.00

17.50

20.00

27.50

40.00

20.00

25.00

28.00

10.00

15.00

20.00

25.00 
PINUS-Continued

- RESINOSA

Tel. Code

Each Per 10

$2 \mathrm{ft} \ldots \ldots \ldots$....... silvery $\ldots . \$ 1.75 \quad \$ 16.00$

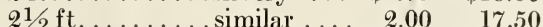

$3 \mathrm{ft} \ldots \ldots \ldots$....... simmered : $\quad 3.00 \quad 27.50$

$4 \mathrm{ft} \ldots \ldots \ldots \operatorname{sim} \ldots \ldots . \quad 4.00 \quad 37.50$

- RIGIDA.

$4 \mathrm{ft} \ldots \ldots \ldots \operatorname{simp} \ldots . .3 .50$

Pitch Pine $5 \mathrm{ft} \ldots \ldots \ldots \ldots$ simmering. 4.50

- STROBUS ............... 2- $3 \mathrm{ft} \ldots \ldots \ldots$ simply ... 2.00

White Prne $3-4 \mathrm{ft} \ldots \ldots \ldots \sin \ldots \ldots .2 .75$

$4-5 \mathrm{ft} \ldots \ldots \ldots$ sinner .... 3.75

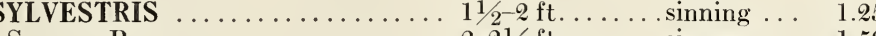

10.00

Scotch PINe

$2-21 / 2 \mathrm{ft} \ldots \ldots$. sincere $\ldots . \quad 1.50$

$21 / 2-3 \mathrm{ft} \ldots \ldots$. . sincerely . . $\quad 1.75$

$3-4 \mathrm{ft} \ldots \ldots \ldots$ sincerity . . $\quad 2.50$

$4-5 \mathrm{ft} . \ldots \ldots$.... sinecure... $\quad 3.50$

RETINOSPORA ERICOIDES.

18 in ......... sinfully ... 1.25

$2 \mathrm{ft} \ldots \ldots \ldots$....... singer $\ldots .2 .00$

— OBTUSA J JPAN CYPRESS $\ldots \ldots \ldots 18$ in .......... singing $\ldots .2 .00$

$2 \mathrm{ft} \ldots \ldots \ldots$............. 2.75

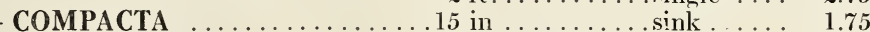

18 in ................. 2.00

$2 \mathrm{ft} \ldots \ldots \ldots$. sipping $\ldots . \quad 2.50$

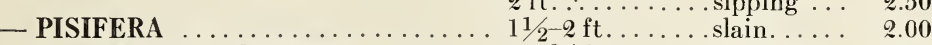

Pea Fruited Cypress $\quad 2-21 / 2 \mathrm{ft} \ldots \ldots \ldots$ slander $\ldots . .2 .50$

$21 / 2^{-3} \mathrm{ft} \ldots \ldots$. slandered . $\quad 3.00$

$3-4 \mathrm{ft} \ldots \ldots$. . slandering $\quad 4.00$

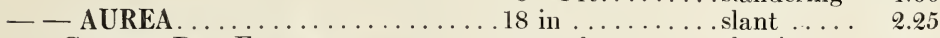

Golden Pea Fruteded $2-3 \mathrm{ft} \ldots \ldots \ldots$. . . slanting .. 3.00

PIUMOSA $3-4 \mathrm{ft} \ldots \ldots$. . slap ...... 4.00

$15-18$ in ........ slave ...... 1.25

Plume-Like Cypress $\quad 11 \frac{1}{2}-2 \mathrm{ft} . \ldots . \ldots$. . slavery $\ldots . .2 .00$

12.00

20.00

27.50

10.00

15.00

$2-21 / 2 \mathrm{ft} \ldots \ldots$. . slavish $\ldots .2 .50 \quad 22.50$

$21 / 2-3 \mathrm{ft} \ldots \ldots$. . slayed $\ldots . .3 .50 \quad 32.50$

$3-4 \mathrm{ft} . . . .$. slayer $\ldots . .4 .00$

$4 \mathrm{ft} \ldots \ldots$..... slay ..... $\quad 6.00$

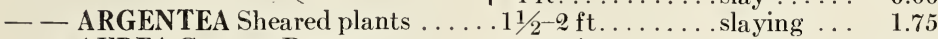

- - AUREA Golden Flumed ...... 15-18 in ....... slender ... 1.25

$1.25 \quad 10.00$

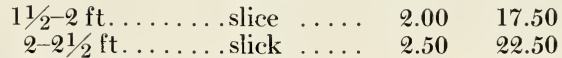

Sheared plants

$21 / 2^{-} 3 \mathrm{ft} \ldots \ldots$. slide $\ldots . . .3 .00$

27.50

- - FILIFERA

$3 \mathrm{ft} \ldots \ldots . . . . . \operatorname{sip} \ldots \ldots . \quad 4.00$

Thread Branched

$11 / 2-2 \mathrm{ft} . \ldots \ldots$ slot $\ldots \ldots, \quad 2.50$

2- $3 \mathrm{ft} \ldots . .$. sloter .... 3.25

- - - AUREA, bushy

$12-15$ in ......... slouchy ... $\quad 2.00$

GOLDEN

10-12 in ...... slowly ... 1.50

12.50

$15-18$ in ....... slug ..... $2.00 \quad 17.50$

- - SQUARROSA VEITCHII.

$11 / 2-2 \mathrm{ft} \ldots \ldots \ldots$ smart ..... 2.25

VeITCh's CyPRESS

$2-21 / 2 \mathrm{ft} \ldots \ldots$. . smartly $\ldots . \quad 2.75$

$21 / 2-3 \mathrm{ft} \ldots \ldots$. . smartness . $\quad 3.50$

32.00

$3-4 \mathrm{ft} \ldots \ldots \ldots$. . . smash ... . 4.50

37.50

- SULPHUREA

$4 \mathrm{ft} \ldots \ldots \ldots$. . . smile .... $\quad 6.00$

RHODODENDRON hardy varieties.

$12-15$ in . . . . . s. smoked ... 1.75

few buds .................15-18 in ...... smoke ... 3.50

few budded. ..................... $11 / 2-2 \mathrm{ft} \ldots \ldots \ldots$.

few budded................ $2-3 \mathrm{ft} \ldots \ldots \ldots$ smuggling $\quad 5.00$

— MAXIMUM, bushy ............ $11 \frac{1}{2} \mathrm{ft} \ldots \ldots \ldots$ soak ..... 2.50

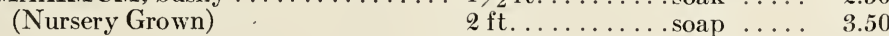

$21 / 2 \mathrm{ft} \ldots \ldots$.... soar $\ldots \ldots . \quad 4.50$

$3 \mathrm{ft} . \ldots \ldots \ldots$.... soaring ... 5.00

SCIADOPITYS VERTICILLATA

$3 \mathrm{ft} \ldots \ldots \ldots$...... socket .... 7.50

Umbrella Pine

$31 / 2 \mathrm{ft} \ldots \ldots$...... sock .... 8.50

$4 \mathrm{ft} \ldots \ldots \ldots \ldots \operatorname{sod} \ldots \ldots .9 .50$

$5 \mathrm{ft} \ldots \ldots \ldots$. soda .... 12.00 


\begin{tabular}{|c|c|c|c|}
\hline & Tel. Code & Each & Per 10 \\
\hline \multirow{4}{*}{$\begin{array}{l}\text { TAXES CUSPIDATA } \\
\text { JAPANESE YEW }\end{array}$} & $.12-15$ in . . . . . song & $\$ 1.75$ & $\$ 16.00$ \\
\hline & $15-18$ in $\ldots \ldots \ldots$ songster & 2.00 & 18.00 \\
\hline & $18-24$ in $\ldots \ldots \ldots$ sonnet $\ldots$ & 2.50 & \\
\hline & $2-21 / 2 \mathrm{ft} . \ldots \ldots$ soon $\ldots \ldots$ & 3.50 & \\
\hline - - BREVIFOLIA & .10-12 in ........ sore . & 1.75 & \\
\hline \multirow{4}{*}{ _- CAPITATA . . } & $.12-15$ in . . . . . spacing ... & 1.75 & 15.00 \\
\hline & $15-18$ in $\ldots \ldots$. space $\ldots$ & 2.50 & 22.00 \\
\hline & 18 in .......... spade & 2.75 & 25.00 \\
\hline & $2 \mathrm{ft} \ldots \ldots \ldots \ldots$ spaded..... & $3.7 \tilde{5}$ & \\
\hline — REPANDENS & $.12-15$ in . . . . . . spangle ... & 1.75 & \\
\hline THUYA OCCIDENTALIS & $18-24$ in $\ldots \ldots$ sparkle $\ldots$ & .75 & 7.00 \\
\hline \multirow[t]{4}{*}{ American Arbor Vitae } & $2-21 / 2 \mathrm{ft} . \ldots \ldots$ special $\ldots$ & 1.25 & 12.00 \\
\hline & $21 / 2-3 \mathrm{ft} . \ldots \ldots$ specify. ... & 1.50 & \\
\hline & $3-4 \mathrm{ft}$. & 2.00 & 17.50 \\
\hline & t- $41 / 2 \mathrm{ft} \ldots \ldots$. speech & 3.50 & \\
\hline - AUREA LUTEA. & $11 / 2 \mathrm{ft} \ldots \ldots \ldots$ speed $\ldots \ldots$ & 1.75 & \\
\hline Geo. Peabody & $2 \mathrm{ft} \ldots \ldots \ldots \ldots$ speeding $\ldots$ & 2.00 & \\
\hline \multirow[t]{2}{*}{ - - BOOTHI COMPACTA } & ....... spine .... & 1.25 & \\
\hline & $11 / 2 \mathrm{ft} \ldots \ldots \ldots$. spinnet & 1.50 & \\
\hline - - COLUMBIAN & $.15-18$ in . . . . . spirit & 1.25 & \\
\hline TIPPED & $11 / 2-2 \mathrm{ft} \ldots \ldots \ldots$ spiritual $\ldots$ & 1.50 & \\
\hline \multirow{3}{*}{$\begin{array}{r}\text { - COMPACTA . } \\
\text { PARSOX'S DWARF }\end{array}$} & .12 in $\ldots \ldots \ldots$. splendor $\ldots$ & 1.25 & 10.00 \\
\hline & 15 in $\ldots$ & 1.75 & 15.00 \\
\hline & $11 / 2 \mathrm{ft} \ldots \ldots \ldots$ sp & 2.00 & \\
\hline - - ELLWANGERIANA & $\begin{array}{r}12-18 \text { in } \ldots \ldots \ldots \text { sponge } \ldots \\
11 / 2 \mathrm{ft} \ldots \ldots \ldots \text { spool } \ldots \ldots\end{array}$ & $\begin{array}{l}1.25 \\
1.75\end{array}$ & $\begin{array}{l}10.00 \\
15.00\end{array}$ \\
\hline —-GLOBOSA .. & .12 in $\ldots \ldots \ldots$.... sporting . . & 1.50 & \\
\hline \multirow{2}{*}{$\begin{array}{l}\text { DWARF GLOBE } \\
\text { - HOVEYI. }\end{array}$} & 18 in $\ldots \ldots \ldots \ldots$ spot & 2.00 & \\
\hline & $12-15$ in . . . . . spreading . & 1.25 & \\
\hline \multirow[t]{2}{*}{ Hover's Goldex DWAR } & $15-18$ in $\ldots \ldots \ldots$ spread & 1.50 & \\
\hline & $18 \mathrm{in}$ & 2.00 & \\
\hline \multirow{4}{*}{ - - PLICATA (trivifolia) } & $.18-24$ in & 1.50 & 12.50 \\
\hline & $2-21 / 2 \mathrm{ft} \ldots \ldots \ldots \mathrm{spu}$ & 1.75 & 15.00 \\
\hline & $21 / 2^{-3} \mathrm{ft} \ldots \ldots \ldots \mathrm{sp}$ & 2.00 & 17.5 \\
\hline & $3 \mathrm{ft} \ldots \ldots \ldots \ldots \mathrm{sp}$ & 3.00 & \\
\hline \multirow{4}{*}{- - PYRAMId ALIS $\ldots . .}$. & $.18-24$ in & 1.00 & 7. \\
\hline & $2-21 / 2 \mathrm{ft} . \ldots \ldots$ square $\ldots$ & 1.75 & 15.00 \\
\hline & $21 / 2-3 \mathrm{ft} \ldots \ldots \ldots$ squarely $\ldots$ & 2.00 & 18.00 \\
\hline & $3-4 \mathrm{ft} \ldots \ldots \ldots \mathrm{sc}$ & $2.7 j$ & 25.00 \\
\hline & $4-5 \mathrm{ft} \ldots \ldots \ldots \mathrm{sc}$ & 3.50 & \\
\hline \multirow{2}{*}{ - - ROSENTHALI . } & $11 / 2 \mathrm{ft} \ldots \ldots \ldots$ st & 1.75 & 15.00 \\
\hline & $2 \mathrm{ft} \ldots \ldots \ldots \ldots \mathrm{st}$ & 2.00 & \\
\hline \multirow{2}{*}{ _- SPAETHII } & $\mathrm{n} \ldots \ldots \ldots$ st & 1.00 & 7.50 \\
\hline & ......st & 1.25 & 10.00 \\
\hline \multirow{3}{*}{$\begin{array}{c}\text { - UMBRACULIFERA } \\
\text { DWARF ARBOR VITAE }\end{array}$} & $n \ldots \ldots$....st & 1.50 & 12.00 \\
\hline & n ....... stammer .. & 1.75 & 15.00 \\
\hline & $\ldots \ldots s$ & 2.75 & \\
\hline \multirow[t]{4}{*}{ —- VERVAENEANA } & $11 / 2 \mathrm{ft}$. & 1.75 & \\
\hline & $2 \mathrm{ft} \ldots \ldots \ldots$ standard . & 2.00 & \\
\hline & $21 / 2 \mathrm{ft}$. & 2.75 & \\
\hline & ......starch & 4.00 & \\
\hline \multirow{2}{*}{ FIBERIAN } & .18 in . . . . . . sta & 1.25 & \\
\hline & ..sta & 1.75 & \\
\hline VADENSIS & $2-21$ & 2.50 & \\
\hline Hemlock Sprdce & $21 / 2-3 \mathrm{ft} \ldots \ldots$. statement & 3.50 & \\
\hline & te $\ldots$ & 4.50 & \\
\hline & $4-5$ & 6.00 & \\
\hline - CAROLINA. & $11 / 2 \mathrm{ft} \ldots \ldots$ & 3.50 & \\
\hline Carolina Hemlock & ... steak ..... & 3.75 & \\
\hline & $\ldots \ldots s$ & 4.00 & \\
\hline & $\ldots \ldots$ & 5.50 & \\
\hline CCA F & ......st & .35 & 3.00 \\
\hline ADAM's NEEDI & .......steward .. & .50 & 4.0 \\
\hline
\end{tabular}




\section{HARDY HERBACEOUS PERENNIALS}

All of these are field grown plants, nice strong clumps depending upon the variety. 25 c each $-\$ 2.00$ per $10-$ except where noted.

Tel. Code

ACHILLAE TOMENTOSA

oasis ....

AJUGA REPTANS

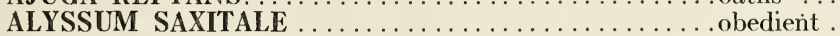

ANCHUSA ITALICA $\ldots \ldots \ldots \ldots \ldots \ldots \ldots \ldots \ldots \ldots \ldots \ldots \ldots$ obediently

ANEMONE JAPONICA, pink and red .............. obey ....

ANTHEMIS TINCTORIA, KELWAYI ............. object ....

AQUILEGIA CHRYSANTHA ................. obejctive

ARABIS ALPINA .........................

ARENARIA CASPITOSA $\ldots \ldots \ldots \ldots \ldots \ldots \ldots \ldots \ldots$. $\ldots$ bligate

ARMERIA FORMOSA $\ldots \ldots \ldots \ldots \ldots \ldots \ldots \ldots \ldots$ obligations

ASTER Robert Parker . . . . . . . . . . . . . . . . obligating

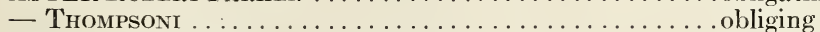

BELLIS PERENNIS . . . . . . . . . . . . . . . . obscene

BOLTONIA LATISQUAMI NANA ............... obscenity

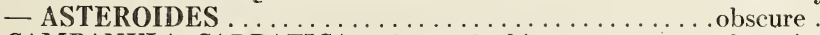

CAMPANULA CARPATICA - blue and white.......... obscurity .

- MEDIA, blue ............................. obscurely

- CALYCANTHEMA - blue, pink, white............. observance

— PERSAECIFOLIA - blue . . . . . . . . . . . . . . . . observant

- PYRAMIDALIS ...................... observe . .

CENTAUREA MONTANA AND RUTHANICA ......... observing .

CERASTIUM TOMENTOSUM . ................... obsolete. .

CHRYSANTHEMUM - Bronze, Boule, Ncige, Donkelaria,

Globe d'Or, St. Iloria, Souv. de Melanie, Tennyson,

Gloria, Jane, Betty and other choice kinds. . . . . . . . . obstacle. . .

- MAXIMUM-Shasta Daisy.................... obstinancy

CONVALARIA-clumps ......................... obstinate.

COREOPSIS LANCEOLATA $\ldots \ldots \ldots \ldots \ldots \ldots \ldots \ldots$ obstinately

DELPHINIUM BELLA DONNA .................... obstruction

- FORMOSUM ........................... obstructive

— KELWAY'S HYBRID MIXED ................. obtaining

- SINENSIS - blue . . . . . . . . . . . . . . . . . obtain

DIANTHUS, DELTOIDES $\ldots \ldots \ldots \ldots \ldots \ldots \ldots \ldots \ldots$ obtained . .

— PLUMARIS . . . . . . . . .

- - Her Majesty, White Reserve $\ldots \ldots \ldots \ldots \ldots \ldots \ldots$. . . . . obvious

FUNKIA COERULEA . . . . . . . . . . . . . . . . ocupy

— SUBCORDATA .................................

- UNDULATA VARIEGATA .................. occupying

GAILLARDIA GRANDIFLORA $\ldots \ldots \ldots \ldots \ldots \ldots \ldots \ldots \ldots$ occurred

SULPHUREA ACULATA ........................ occupancy

GALLIGA HARTLANDI. ...................... occupier .

GEUM ATRO-SANGUINEUM . . . . . . . . . . . . . . . occur

HELENIUM AUTUMNALE SUPERBA . . . . . . . . . . occurring

HELIOPSIS PITCHERIANA $\ldots \ldots \ldots \ldots \ldots \ldots \ldots \ldots \ldots$ oceanic .

HELIANTHUS-Miss Melish $\ldots \ldots \ldots \ldots \ldots \ldots \ldots \ldots \ldots \ldots \ldots \ldots \ldots \ldots$ octagon

- MULTIFLORA FL. PL. . . . . . . . . . . . . . . . octagonal

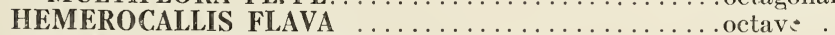

HEUCHERIA SANGUINEA $\ldots \ldots \ldots \ldots \ldots \ldots \ldots \ldots$ odd $\ldots \ldots$

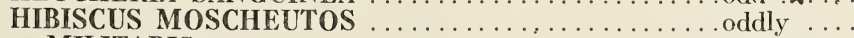

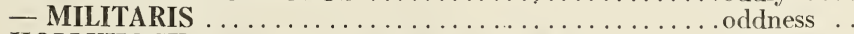

HOLLYHOCK - assorted colors, double and single ....... . ode.....

IRIS CYANEA .............................. offense

- DALMATICA PALLIDA $\ldots \ldots \ldots \ldots \ldots \ldots \ldots \ldots \ldots \ldots \ldots$ offend

— GERMANICA —assorted $\ldots \ldots \ldots \ldots \ldots \ldots \ldots \ldots \ldots \ldots \ldots$ offender

— INTERMEDIA — assorted kinds $\ldots \ldots \ldots \ldots \ldots \ldots \ldots$ offended

— JAPANESE — assorted ..$\ldots \ldots \ldots \ldots \ldots \ldots \ldots \ldots \ldots \ldots$ offending.

- SIBIRICA - Blue and Snow Queen $\ldots \ldots \ldots \ldots \ldots \ldots \ldots$ offensive . .

LATHYRUS-Pink Beauty $\ldots \ldots \ldots \ldots \ldots \ldots \ldots \ldots$ offer $\ldots \ldots$ 
LUPINUS-White

Tel. Code

official

VISCARA CHALEDONICA

officially .

- VISCARA SPLENDENS officiate .

MONARDA DIDIMA and FISTLLOSA $\ldots \ldots \ldots \ldots \ldots$

MYOSOTUS PALUSTRIS

OENOTHERA SPECIOSA

oily $\ldots . . .$.

PACHYSANDRA TERMINAiIS $\ldots \ldots \ldots \ldots \ldots \ldots \ldots$ oiled $\ldots \ldots \ldots$

1 yr. pots

ointment

older ......

old $\ldots . .$. .

Each Per 10

PAEONIES OFFICINALIS-assorted

— SINENSIS - Assorted colors, named kinds . .......... omission .

- FESTIVA MAXIMA ...................... omitted .

PAPAVER ORIENTALIS $\ldots \ldots \ldots \ldots \ldots \ldots \ldots$. . . . on . . . .

$\$ .50-\$ 4.50$

$.50 \quad 4.50$

$.65 \quad 5.00$

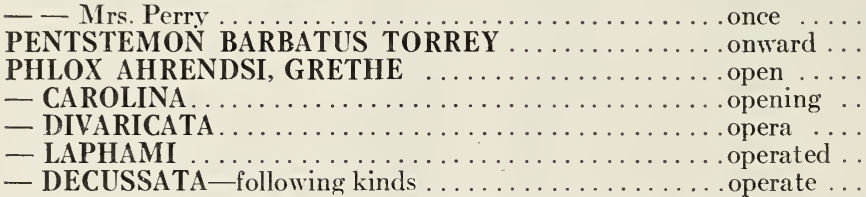

Europea, Anth. Mercier, Beranger, Gen'l van Heutz, Thebaide, La Vogue, Coquelicot, Eclaireur, Matador, M. P. Langier, Sieboldli, Von Lassburg, Independence, Queen.

- Elizabeth Campbell .......................... operation

— Fraulein Krupp......................... opiate ....

- Suffrutocisa, Indian Chief, and Miss Lingard. . . . . . . . opinion ...

- subulata pink and white $\ldots \ldots \ldots \ldots \ldots \ldots \ldots \ldots$. . . . . .

PHYSOSTIGIA — pink ...................... optical ...

PLUMBAGO - larpentae ..................... optional .

POLEMONIUM - coeriulea . . . . . . . . . . . . . . optioned .

PYRETHRUM — single . . . . . . . . . . . . . . . . . . . . . . . ordain .

- grandiflora. nixed ....................... ordained .

RUDBECKIA lacineata. Golden Glow . . . . . . . . . . . . . order ....

SALVIA - azurea ............................. ordinary .

SATURA MONTANA . . . . . . . . . . . . . . .

SEDUM - Spectabiles . . . . . . . . . . . . . . . . . originate

STACHYSLYANATA … . . . .

STOKESIA, blue. ....

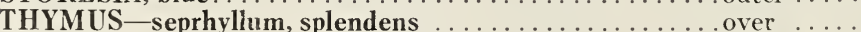

TRITOMA - uvaria . . . . . . . . . . . . . . . . overturn

- PFITZERI ..........................................

TUNICA !axifraga $\ldots \ldots \ldots \ldots \ldots \ldots \ldots \ldots \ldots \ldots \ldots \ldots \ldots$ overflow

VALERIAN ............................ overtake

VERONICA - spicata ..................................

VINCA -minor (per thousand $\$ 60.00) \ldots \ldots \ldots \ldots \ldots$ ow $\ldots \ldots \ldots$

VIOLA-Papillon, dark blue . . . . . . . . . . . . . . oyster ...

\section{HARDY GRASSES}

Tel. Code Each Per 10

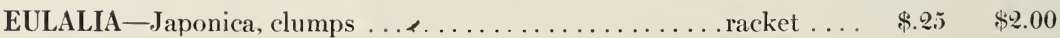

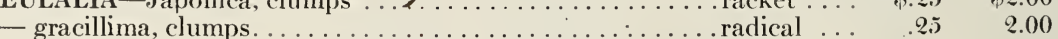

- variegated, clumps $\ldots \ldots \ldots \ldots \ldots \ldots \ldots \ldots \ldots \ldots \ldots \ldots \ldots$ raft $\ldots \ldots \ldots .2 .5 \quad 2.00$

FESTUCA -

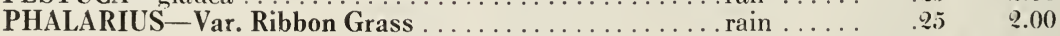




\section{FRUIT DEPARTMENT}

Tel. Code Each

APPLES, Leading Kinds-Extra, $11 / 8$ in .............. XX $\$ 2.00$

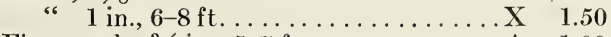

First grade, $3 / 4$ in., $5-7 \mathrm{ft} \ldots \ldots \ldots \ldots$. A $\quad 1.00$

DWARF APPLES-4-5 ft., 1 in., extra. . . . . . . . . . . . . . 1.50

$3-4 \mathrm{ft} ., 3 / 4$ in., first class. . . . . . . . . . . . . . 1.00

PEAR, Leading Kinds-Std., $1 \frac{1}{8}$ in., $6-8 \mathrm{ft} \ldots \ldots \ldots \ldots \ldots \ldots \ldots \times \ldots$

Extra, $7 / 8$ and 1 in., $6-7 \mathrm{ft} \ldots \ldots \ldots \ldots \ldots . . . .21 .50$

First Grade, $3 / 4-7 / 8$ in., $5-7 \mathrm{ft} \ldots \ldots \ldots \ldots \ldots$. 1.00

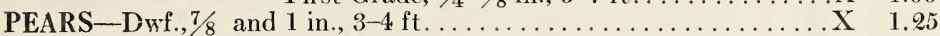

First Grade, $3 / 4^{-7} / 8$ in., $3-4 \mathrm{ft} \ldots \ldots \ldots \ldots \ldots \ldots \ldots$ A 1.00

Med. grade, $1 / 2-7 / 8$ in., $2-3 \mathrm{ft} \ldots \ldots \ldots \ldots \ldots \ldots \ldots, \quad .75$

$\begin{array}{r}\text { CHERRIES-Sour, Leading Kinds } \\ \text { Extra, } 1 \text { in., } 6-7 \mathrm{ft} \ldots \ldots \ldots \ldots \ldots \ldots \ldots \ldots \ldots \ldots \ldots \ldots \\ \hline\end{array}$

$\begin{array}{lll}\text { Extra, } 1 \text { in., } 6-7 \mathrm{ft} \ldots \ldots \ldots \ldots \ldots \ldots \ldots \ldots \ldots \ldots \ldots \ldots \ldots \ldots & 1.75 \\ \text { First Grade, } 3 / 4-7 / 8 \text { in., } 5-7 \mathrm{ft} \ldots \ldots \ldots \ldots \ldots \ldots \ldots \ldots & 1.50\end{array}$

Med. grade, $5 / 8-3 / 4$ in., $4-5 \mathrm{ft} \ldots \ldots \ldots \ldots \ldots \ldots \ldots$ B 1.25
CHERRIES-Sweet, Leading Kinds

Extra, 1 in., $6-7 \mathrm{ft} \ldots \ldots \ldots \ldots \ldots \ldots \ldots \ldots \ldots \ldots \ldots \ldots$

First grade, $3 / 4-7 / 8$ in., $5-7 \mathrm{ft} \ldots \ldots \ldots \ldots \ldots \ldots \ldots$ A 1.50

Med. grade, $5 / 8-3 / 4$ in., $4-5 \mathrm{ft} \ldots \ldots \ldots \ldots \ldots \ldots \ldots$. . . . 1.25

PLUMS, Leading Kinds $-11 / 4$ in.. $6-8 \mathrm{ft} \ldots \ldots \ldots \ldots \ldots \ldots \ldots \ldots \ldots$

Extra, $7 / 8$ and 1 in., $5-7 \mathrm{ft} \ldots \ldots \ldots \ldots \ldots \ldots \ldots \ldots$

First grade, $3 / 4-7 / 8$ in., $5-7 \mathrm{ft} \ldots \ldots \ldots \ldots \ldots \ldots \ldots \ldots$. 1.25

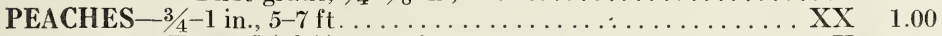

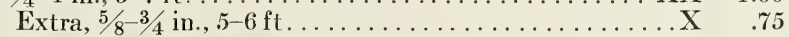

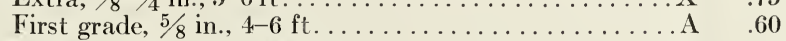

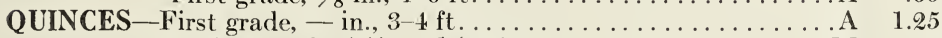

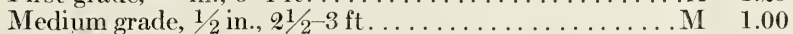

NUTS -Chestnut American, $4-6 \mathrm{ft} \ldots \ldots \ldots \ldots \ldots \ldots \ldots \ldots \ldots \ldots$

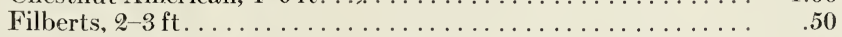

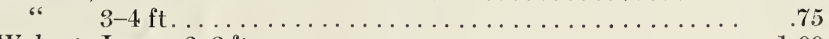

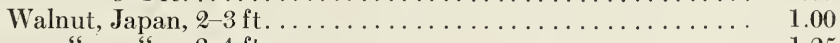

$3-4 \mathrm{ft} \ldots \ldots \ldots \ldots \ldots \ldots \ldots \ldots \ldots \ldots \ldots \ldots \ldots \ldots \ldots, 1.25$

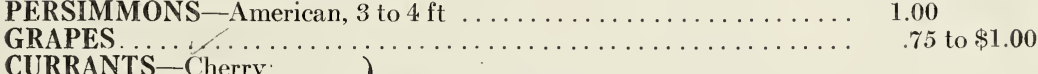

Fay's Prolific $\}$ Medium, 2 years ............. .50

White Grape $\}$ Strong, 2 years. . . . . . . . . . . . . .

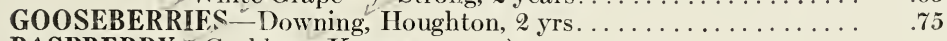

$\left.\begin{array}{r}\text { RASPBERRY } \\ \text { - Cu hbert, Kansas } \\ \text { St. Regis, (everbearing) }\end{array}\right\} \ldots \ldots \ldots \ldots \ldots \ldots \ldots \ldots .20 \quad \$ 2.00$ per doz.

BLACKBERRIES

$\begin{array}{lr}\text { St. Regis, (everbearing) }\{\ldots \ldots \ldots \ldots \ldots \ldots \ldots \ldots & .20 \\ .20\end{array}$

STRAWBERRIES - pot plants...................

ASPARAGUS - Con. Colossal, a yrs ................ per hundred.

RHUBARB.

Special prices will be quoted on all large fruit lists.

$20 \$ 2.00$ per doz.

8.00
2.50

.25 
